Article

\title{
Optimizing Xylindein from Chlorociboria spp. for (Opto)electronic Applications
}

\author{
R.C. Van Court ${ }^{1}{ }^{1}$, Gregory Giesbers ${ }^{2}$, Oksana Ostroverkhova ${ }^{2}\left(\mathbb{D}\right.$ and Seri C. Robinson ${ }^{1, *}$ \\ 1 Department of Wood Science and Engineering, Oregon State University, Corvallis, OR 97331, USA; \\ ray.vancourt@oregonstate.edu \\ 2 Department of Physics, Oregon State University, Corvallis, OR 97331, USA; giesberg@oregonstate.edu (G.G.); \\ ostroveo@science.oregonstate.edu (O.O.) \\ * Correspondence: seri.robinson@oregonstate.edu
}

Received: 26 October 2020; Accepted: 12 November 2020; Published: 17 November 2020

\begin{abstract}
Xylindein, a stable quinonic blue-green fungal pigment, has shown potential for use not only as a colorant but also as an (opto)electronic material. As no method presently exists to synthesize the pigment, organic production by slow-growing fungi from the genus Chlorociboria is the only method to obtain it. This has resulted in limited quantities of impure xylindein, hampering research. In order to improve quantity and quality of pigment for optoelectronic applications, speed of xylindein production by Chlorociboria aeruginosa and its relative purity were compared across liquid and solid-state fermentation conditions on selected nutrient sources. Liquid $2 \%$ malt shaking cultures produced the same amount of pigment in 5 weeks that previous testing produced in 2 months. Xylindein generation speed, purity, and conductive properties of produced pigment for (opto)electronics was then compared between two Chlorociboria species native to North America, Chlorociboria aeruginosa and Chlorociboria aeruginascens. Differences were seen in the conductivity of extracted pigment between species and strains, with xylindein from $C$. aeruginascens strain UAMH 7614 producing films with the highest effective electron mobility. The identification of the most effective growth conditions and the strain with highest purity xylindein production should support further development of sustainable organic (opto)electronics. Future work identifying new strains with reduced production of interfering metabolites and new extraction methodologies will help to produce very low cost xylindein, supporting sustainable technologies based on the pigment.
\end{abstract}

Keywords: spalting; Chlorociboria; organic semiconductor; (opto)electronics; fungal pigment; secondary metabolites; green energy

\section{Introduction}

The pigment xylindein is a blue-green quinonic secondary metabolite produced by soft rot decay fungi within the genus Chlorociboria. Wood colored by Chlorociboria has a long history of use in art, with pieces dating back to the 15th century retaining their blue-green coloration today [1,2]. In modern times, pigment extracted from Chlorociboria spp. has been investigated for use as a coloration agent for wood [3], for paints [4], and for dying textiles [5-9]. In addition, xylindein has unique physical properties that are of interest to the field of optoelectronics. Xylindein possesses a $\pi$-conjugated core structure and exhibits aggregate formation likely due to hydrogen bonding and molecular $\pi-\pi$ stacking [10]. This enables electron transport with mobility of up to $-0.4 \mathrm{~cm}^{2} /(\mathrm{Vs})$ [11] in amorphous xylindein films and potentially higher in high-purity [12] and/or crystalline xylindein. The observed semiconducting properties, in addition to excellent photostability of xylindein, make it a candidate for green technologies such as sustainable organic electronics [10-13]. 
Despite multiple investigations into laboratory synthesis of xylindein, none have thus far been successful [14-16]. This makes synthesis by Chlorociboria spp. The only way to obtain the pigment. Unfortunately, the fungi grow slowly under most laboratory conditions [17] and can lose their pigmentation in culture $[18,19]$. Research into the relationship between culture conditions and pigment production of Chlorociboria cultures has been the subject of multiple investigations (Table S1). The first was in 1928, where growth substrates such as beech wood and organic medias such as malt extract and plum decoction were found to result in blue-green pigmentation [20]. Later work identified sugar-based media as showing the best pigmentation, with organic nitrogen sources inhibiting pigmentation [19]. Recent work has suggested that this effect may be due to nitrogen limitation leading to stimulation of pigment production, with high available nitrogen growth conditions leading to high biomass production but no pigmentation [21].

The standard methodology of acquiring xylindein for present day (opto)electronic research has relied upon use of $2 \%$ malt agar plates amended with Acer saccharum (Marshall) woodchips. This media has been found to increase colony size and improve pigmentation across Chlorociboria aeruginascens isolates [17,22]. It also allows for straightforward extraction of the pigment into a solvent [23]. However, recent work has also shown that liquid media growth conditions also produce strong pigmentation results [24-26] and may be easier to scale for industrial production.

While multiple publications have studied pigmentation in Chlorociboria cultures, methodologies have been highly varied and results have been difficult if not impossible to directly compare. They have ranged from morphological descriptions to extracted pigment color (Table S1). No studies have compared colony diameter to extractable pigment amounts, or directly compared xylindein production between liquid and solid medias. This makes it difficult to identify which tested growth conditions actually result in the best pigment production. In addition, it is unknown which growth conditions result in the purest extracted xylindein solution. Current extraction methodology from xylindein does not separate it from a variety of other metabolites that have been reported from Chlorociboria cultures extracted into dichloromethane [24], or components of media. These contaminants have been found to interfere with the electronic potential of xylindein, reducing conduction [12]. No work has been performed to investigate which growth conditions are associated with more impurities. Previous work on purification of the pigment used centrifugal partition chromatography [27]; however, specialized equipment was required and only $70 \%$ extraction efficiency was achieved.

In addition to variation in pigment production in response to environmental conditions, production differences between fungal species and strains may also be significant. In North America, two species in the Chlorociboria genus appear sympatrically, Chlorociboria aeruginascens and the slightly less common Chlorociboria aeruginosa [18]. The two species are morphologically similar although genetically quite distinct, both showing more genetic similarities to southern hemisphere Chlorociboria species than to each other $[22,28]$. The primary physical differences between the two species are that C. aeruginascens has smaller spores and smooth instead of rough tomentum hyphae [18,29].

Different species of pigmenting fungi within the same genus are known to show variation in pigment production. Notably, two pigmenting spalting fungi, Scytalidium cuboideum and Scytalidium ganodermophthorum, show very different pigmentation patterns, with S. cuboideum producing a naphthoquinonic red pigment [30] and S. ganodermophthorum producing an unknown yellow pigment $[8,31]$. However, production of blue-green pigment is conserved within the Chlorociboria genus [32], although variation in pigment production between species and strain has been noted [22,24].

In addition to growth speed differences between species, there is also a high likelihood of secondary metabolite production variation between species and strains. Variation in pigmentation between fungal strains is well known. For example, strains of Monascus purpureus have shown variation in production of pigment and toxic secondary metabolite citrinin [33], and multiple mutants produce variations in pigmentation $[34,35]$. Chlorociboria species produce other secondary metabolites including a yellow compound [24], which, in addition to other metabolites from the fungi, may be responsible for reducing the conductivity of films made from extracted xylindein [12]. Pigment extracted from 
fungi shows a great deal of variability, as they are biological organisms that respond differently to their environments. The inhomogeneity associated with biological production is an obstacle to research into (opto)electronics, where purity and consistency are desired. Xylindein extract from one fungus or growth condition may have a different profile of secondary metabolites that interfere with conductivity, complicating interpretations of results into (opto)electronic applications. However, no previous research has been conducted comparing xylindein quality from different sources. Identification of the pigment source with best (opto)electronic capabilities is therefore important to support further research into technologies based on the pigment. Minimization of these interfering metabolites should also simplify purification methodologies, allowing for cheaper mass production of purified pigment for optoelectronic technologies.

This research sought to identify Chlorociboria spp. growth conditions for the highest xylindein production with the fewest contaminants. This will support further research into xylindein's use in (opto)electronics. Sustainable, natural product-derived materials such as xylindein have been a focus of research for organic electronics [36,37]. Organic semiconductors have economic and material advantages for photovoltaics in addition to their sustainability [38,39]. Pigments in particular have been shown to be effective organic semiconductors [40-44]. Identification of growth conditions that result in pigment extracts with purity high enough to support direct use in (opto)electronics could allow for low-cost production of technologies built on xylindein. As an organic optoelectronic material with good light stability [13] it could be used in devices such as organic solar cells, which have the potential to replace the environmental issues associated with traditional solar cell technology [45]. In order to obtain quality material to support this research, the systemic comparison of existing production methods to new processes is critical to establish the best growth conditions. Tailoring production of secondary metabolites from fungi for (opto)electronics is a novel approach in the field of organic semiconductors.

Pigment production was compared in multiple media across liquid shaking, liquid static, and solid woodchip-amended plate cultures, and parallels were drawn with all previous stimulatory Chlorociboria growth/pigment generation research (the two are often interchangeable in publications despite not meaning the same thing). Next, to compare speed, quantity, and quality of xylindein production, this study compared growth of two C. aeruginascens strains (UAMH 11655 and UAMH 7614) and one C. aeruginosa strain (UAMH 11657)—all commonly used in U.S. spalting research. Identification of the species and strain of Chlorociboria that produces the highest extractable quantities of the pigment xylindein, with best purity, in the quickest time, will allow for the most efficient production of the pigment. This budding technology could allow for development of truly sustainable energy production, and identification of "best" species and strain (in terms of xylindein synthesis) is critical for expansions of research, purification studies, and technological adoption of xylindein into commercial green energy fields.

\section{Materials and Methods}

\subsection{Preliminary Media Screen}

Chlorociboria aeruginosa (UAMH 11657, isolated from a rotting hardwood log in Halliburton, ON, Canada) was inoculated into $50 \mathrm{~mL}$ of sterilized media in $250 \mathrm{~mL}$ borosilicate Mason jars with plastic screw top lids. All media were inoculated using single point inoculation (6 $\mathrm{mm}$ diameter round) from cultures grown on $2 \%$ malt agar, sealed with Parafilm "M" and allowed to grow at ambient room conditions $\left(21^{\circ} \mathrm{C}\right.$ and $35 \%$ relative humidity (RH)) for $2,3,5$, or 23 weeks with a total of 5 repetitions of each tested condition. Mediums tested included Czapek Dox Liquid Medium (M1170A, HIMEDIA, Mumbai, India), low pH fungal broth (M265, HIMEDIA, Mumbai, India), potato dextrose broth (254920, Difco, Franklin Lakes, NJ, USA), 2\% brewer's malt extract (Briess CBW Sparkling Amber Dry Malt Extract, Chilton, WI, USA), 2\% bacteriological malt extract (VWRVJ873, VWR Life Science, Radnor, PA, USA), 2\% honey (Glory Bee Raw Pacific Northwest Blackberry Blossom Honey, Eugene, OR, 
USA), $2 \%$ of $10.4 \%$ cellulose nanocrystal suspension (University of Maine Process Development Center, ME, USA), peptone yeast glucose (PYG) media (30 g/L dextrose (BDH9230, VWR Analytical, Radnor, PA, USA), $1.25 \mathrm{~g} / \mathrm{L}$ ultrapure bacteriological peptone (J20048-P2, Thermo Scientific, Waltham, MA, USA), $1.25 \mathrm{~g} / \mathrm{L}$ yeast extract(103303, Mp Biomedicals, China)), yeast peptone soluble starch (YPSS) media (4 g/L yeast extract(103303, Mp Biomedicals, China), $15 \mathrm{~g} / \mathrm{L}$ solubilized starch (470302-754, Ward's Science, Henrietta, NY, USA), $1 \mathrm{~g} / \mathrm{L} \mathrm{K}_{2} \mathrm{HPO}_{4}(0781$, VWR Life Science, Radnor, PA, USA), $0.5 \mathrm{~g} / \mathrm{L} \mathrm{MgSO}_{4}(\mathrm{BDH} 9246$, VWR Analytical, Radnor, PA, USA)). Media for further experimentation were selected at week 5 on the basis of growth morphology, although the experiment was continued until week 23 to see if extracellular secretion of pigment would occur. Bacteriological malt, brewer's malt, potato dextrose broth (PDB), and honey were selected for further testing, as these media showed pigment production in hyphae by week 5 of growth.

\subsection{Growth Conditions Testing}

Chlorociboria aeruginosa growth was compared across solid-state fermentation, static liquid state fermentation, and shaking liquid state fermentation states for 4 media selected on the basis of preliminary results. Bacteriological malt, brewer's malt, and honey were all analyzed for main sugars by the Linus Pauling Core Laboratories at Oregon State University, with the following results: bacteriological malt showed $16.1 \%$ glucose, $3.22 \%$ fructose, $76.93 \%$ maltose, and $3.65 \%$ sucrose; brewer's malt showed $18.12 \%$ glucose, $2.19 \%$ fructose, $73.35 \%$ maltose, and $6.33 \%$ sucrose; and honey contained $53.75 \%$ glucose, $41.10 \%$ fructose, $4.57 \%$ maltose, and $0.59 \%$ sucrose. Liquid media cultures were grown and extracted following the general method laid out in Weber et al. (2016), and solid cultures followed the general method laid out in Robinson et al. (2012).

Single point inoculation of Chlorociboria aeruginosa (strain UAMH 11657, isolated from a rotting hardwood log in Halliburton, ON, Canada) was used for all samples. All media was inoculated from cultures grown on $2 \%$ malt agar for 6 weeks at ambient room conditions $\left(21^{\circ} \mathrm{C}\right.$ and $\left.35 \% \mathrm{RH}\right)$. Cultures were then sealed with Parafilm " $\mathrm{M}$ " and allowed to grow at ambient room conditions for 3, 5, 7, or 9 weeks with a total of 5 repetitions of each tested condition. Cultures were then extracted into $30 \mathrm{~mL}$ of HPLC grade dichloromethane (DCM) (BDH23373, VWR, Radnor, PA) and color read, calculating color difference between all samples and dichloromethane alone.

Due to a lack of any visible pigmentation, static brewer's malt and potato dextrose broth (PDB) in week 3 and static PDB in week 5 were ascribed a $\triangle E$ value of zero. Before inoculation and at week 9 , liquid cultures were tested with $\mathrm{pH}$ strip (VWR pH-Test 0-14, BDH35309, VWR, Radnor, PA, USA) to identify $\mathrm{pH}$.

In addition to comparison of extracted pigment production, C. aeruginosa growth was also compared between plates amended with woodchips and those with only tested media. Pigment cannot be extracted from non-amended plates following the standard procedure laid out by [23], but the growth radius was measured from point of inoculation to colony edge, and growth morphologies were compared.

\subsubsection{Liquid Shaking}

Inoculated $250 \mathrm{~mL}$ borosilicate Mason jars containing $50 \mathrm{~mL}$ of sterile media were placed on a shake table (Orbital Gene Scientific Industries, Bohemia, NY, USA) and shaken at $99 \mathrm{rpm}$ for the incubation period. This speed was selected as the fastest speed that would securely support the moving weight of shaking cultures. Contents of the jars were individually blended on the ice crush setting for 2 min with an Oster classic series blender (Oster, Atlanta, GA, USA) to break up hyphae and improve pigment yield, as described in Weber et al. (2016). Blended liquid and $30 \mathrm{~mL}$ of DCM was added per $50 \mathrm{~mL}$ jar of media in a $250 \mathrm{~mL}$ Erlenmeyer flask. Flasks were corked and manually swirled for $3 \mathrm{~min}$ to fully mix water and DCM layers, then allowed to settle for $27 \mathrm{~min}$. Contents of flasks were then placed in a $250 \mathrm{~mL}$ separatory funnel and DCM layer was collected. DCM layer was then color read as described below. 


\subsubsection{Liquid Static}

Inoculated $250 \mathrm{~mL}$ borosilicate Mason jars containing $50 \mathrm{~mL}$ of sterile media were grown statically for set incubation period. Cultures were then blended and extracted, and pigment production was measured via color reading as described for liquid shaking cultures.

\subsubsection{Solid State Woodchip Plates}

Chlorociboria aeruginosa was cultured on sterile disposable Petri plates for each tested media using methodology laid out in Robinson et al. [17]. Each tested liquid media was made up with the addition of sterilized $15 \mathrm{~g}$ bacteriological agar (J637, VWR Life Science, Radnor, PA, USA) per $1 \mathrm{~L}$ of deionized water, and amended with sterilized white-rotted sugar maple (Acer saccharum Marsh.) shavings processed through \#20 mesh in a Wiley mill. Cultures were allowed to grow at ambient room conditions $\left(21^{\circ} \mathrm{C}\right.$ and $35 \% \mathrm{RH}$ ) for incubation period, then were opened, lids were removed, and the cultures were allowed to dry under ambient conditions in a fume hood for $48 \mathrm{~h}$ prior to extraction. Once dry, cultures were broken up into small $(\approx 1 \mathrm{~mm})$ pieces using a blender on the ice crush setting for $2 \mathrm{~min}$ in order to break down hyphal walls and release intra-cellular pigment in addition to extra-cellular pigment already present in media. Ground culture material was placed in a $250 \mathrm{~mL}$ Erlenmeyer flask with $30 \mathrm{~mL}$ of dichloromethane $(\mathrm{DCM})$, and a $7.0 \mathrm{~mm} \times 24.5 \mathrm{~mm}$ magnetic stir bar was added. A rubber stopper was placed on top of the flask to prevent evaporation, and the flask was stirred at $230 \mathrm{rpm}$ for $30 \mathrm{~min}$. After stirring, the solution was filtered through laboratory grade Whatman no. 1002150 (Whatman, Madistone, UK) filter paper and color read as described below.

\subsection{Analysis of Pigment Concentration for Growth Conditions Testing}

A glimmix model followed by Tukey's Honestly Significant Difference (HSD) test was performed on SAS version 9.4, using the independent variables media, week, and growth condition (shaking, static, or woodchip plates) and the dependent variable color difference $(\Delta \mathrm{E})$. Color readings of controls extracted into $\mathrm{DCM}$ were subtracted from $\triangle \mathrm{E}$ values to account for variation in pigmentation resulting from the different media. To account for heteroscedasticity of data, we relaxed the assumption of constant variance for both media and week variables using the random option.

\subsection{Comparisons of Species and Strain Pigmentation}

Fungal cultures were grown in liquid shaking media and on woodchip-amended plates (specific protocols below). Single point inoculation from cultures grown on $2 \%$ malt agar was used, including Chlorociboria aeruginosa (UAMH 11657, isolated from a rotting hardwood log in Halliburton, ON, Canada) and two strains of Chlorociboria aeruginascens, UAMH 11655 (derived from rotting hardwood in Halliburton, ON, Canada) and UAMH7614 (isolated from single ascospore in Lake District, UK). After inoculation, plates and jars were sealed with Parafilm " $\mathrm{M}^{\prime}$ " and allowed to grow at ambient room conditions $\left(21{ }^{\circ} \mathrm{C}\right.$ and $35 \% \mathrm{RH}$, grown in the dark) for 1 to 9 weeks, with 3 cultures from each test condition harvested and extracted each week. Cultures were then extracted into $30 \mathrm{~mL}$ of HPLC-grade dichloromethane (DCM) (VWR BDH23373) and color read as described below, calculating color difference between all samples and dichloromethane alone. In addition, colony radius was measured for each species in woodchip plates via the colony edge, identifying largest diameter going through colony center and averaging this value with smallest diameter for total colony diameter.

\subsubsection{Liquid Shaking Cultures for Strain Comparison}

Liquid shaking cultures were prepared for strain comparison as described above. However, after blending culture liquid and $30 \mathrm{~mL}$ of dichloromethane (DCM) (BDH23373) was stirred at $\approx 100 \mathrm{rpm}$ on a 15 point multiposition stir plate (Variomag Telesystem, Thermo Scientific) for 30 min using a $2 \mathrm{~cm} \times$ $7 \mathrm{~mm}$ stir bar. Contents of flasks were then placed in a $250 \mathrm{~mL}$ separatory funnel and the DCM layer was collected. This layer was then color read as described below. 


\subsubsection{Solid State Woodchip Plates for Strain Comparison}

Woodchip plates with 2\% bacteriological malt extract (VWR Life Science VJ873) amended with white-rotted sugar maple (Acer saccharum Marsh.) were made up, inoculated, and extracted using methods described above for media comparisons. Extracted pigment was color read as described below.

\subsection{Data Analysis for Comparisons of Species and Strain}

A glimmix model followed by Tukey's HSD test was performed on SAS version 9.4, using the independent variables species, growth condition, and week, and dependent variable color difference $(\Delta \mathrm{E})$. To account for heteroscedasticity of data, the assumption of constant variance was relaxed for species and week variables using the random option. The tested hypothesis was that there would be a significant difference between color differences $(\Delta \mathrm{E})$ as a result of the effects of species and week.

An additional glimmix model with Tukey's HSD was run, comparing radius of colony growth, with independent variables week and species. Assumption of constant variance was relaxed using the random option for variable week. The tested hypothesis was that there would be a significant difference between colony radii as a result of the effects of species and week.

\subsection{Color Reading and Difference Calculation}

Color reading was carried out using a Konica Minolta CR-5 Chroma meter, following standard methodology using the CIE L*a*b color space, which measures color in three-dimensional space with an axis running from green $(-a)$ to red $(a)$, another running from blue $(-b)$ to yellow $(+b)$, and a vertical axis $\left(L^{*}\right)$ running from black $(0)$ to white (100) [42]. Color difference $(\Delta \mathrm{E})$ was calculated with the CIE76 formula (Equation (1), below), which is more suitable for liquids than CIE00 and has been used in previous studies as an indirect measure of pigment concentration for spalting fungal pigments $[10,38,40,43]$. The validity of this method has also been confirmed in comparison to HPLC data [22]. Extracted pigment samples were individually color read in $3 \mathrm{~mL}$ VWR Spectrophotometer Cell (414004-067).

$$
\Delta \mathrm{E}=\sqrt{\left(L_{2}^{*}-L_{1}^{*}\right)^{2}+\left(a_{2}^{*}-a_{1}^{*}\right)^{2}+\left(b_{2}^{*}-b_{1}^{*}\right)^{2}}
$$

\subsection{Optical Absorption of Extracted Pigment Solutions}

After color reading data was taken, optical absorption spectra were measured using a Xe lamp (96000 Oriel, Stratford, CT, USA) and a fiber coupled Ocean Optics USB2000 spectrometer (Ocean Optics, Largo, FL, USA) to determine relative purity. Absorbance (A) was calculated from the incident $\left(\mathrm{I}_{0}\right)$ and transmitted (I) beam intensities as $\mathrm{A}=-\log (\mathrm{I} / \mathrm{I} 0)$, then normalized at the peak absorbance around $670 \mathrm{~nm}$. Reflection losses were taken into account by referencing with respect to a cuvette with pure DCM. For comparisons of growth conditions, we dropped PDA samples from comparison due to lack of pigmentation, and a control of ethanol-washed xylindein was included, prepared following methods used in a previous study investigating xylindein purity [12].

\subsection{Measurement of Electronic Properties}

For measurements of current-voltage (I-V) characteristics, voltage was applied to the samples as described in our previous publications $[14,15]$ using a Keithley 237 source-measure unit. Current was measured as a function of applied voltage in the $0-300 \mathrm{~V}$ range, under ambient conditions in the dark. Samples of each tested strain grown to 12 weeks in liquid cultures and extracted as described above were tested before and after an ethanol wash, which has been shown to improve conductivity in previous work [14]. This wash consisted of rinsing vials of solid xylindein adhered to the vial with multiple passes of clean ethanol (Sigma-Aldrich) until the ethanol wash [14] no longer showed measurable fluorescence under UV light. Xylindein powder was then dissolved in DCM to form $10 \mathrm{mg} / \mathrm{mL}$ solutions, which were drop-cast onto glass substrates with interdigitated Au electrodes with 
a $25 \mu \mathrm{m}$ gap. Substrates were patterned using conventional lift-off photolithography, and Au with a $5 \mathrm{~nm} \mathrm{Cr}$ adhesion layer was deposited using a Veeco 7700 thermal evaporator.

The effective mobilities were calculated from the space-charge limited current (SCLC) regime of the I-V characteristics (when I is proportional to the voltage squared $V^{2}$ ), in the thin-film approximation [14,15] (Equation (2)).

$$
j=\frac{2 \mu_{e f f} \varepsilon \varepsilon_{0}}{\pi} \frac{V^{2}}{L^{2}}
$$

where $j$ is the linear current density, $j=\mathrm{I} / d$; I is the measured current; and $d$ is the length of the electrode. $V$ is the applied voltage, $L$ is the gap between the electrodes, $\varepsilon_{0}$ is the vacuum permittivity, $\varepsilon$ is the dielectric constant (assumed to be equal to 3 ), and $\mu_{e f f}$ is the effective mobility.

\section{Results and Discussion}

\subsection{Pigment Variation across Growth Conditions}

Pigment production varied across growth conditions and between species. In the first phase of the experiment, $C$. aeruginosa UAMH 11657 showed different pigment production in response to growth conditions. This was apparent in culture morphology (Figure 1) and significant differences in colony radii (Figure S1). Chlorociboria aeruginosa grown in lab-grade malt extract under shaking conditions resulted in the highest mean extracted pigment production, as measured by color difference $(\Delta \mathrm{E})$, in the shortest amount of time (Figure 2). The interaction between media, week, and growth conditions was highly significant $(\mathrm{df}=(18,150), F$-value $=13.47, p<0.0001)$. However, while shaking lab grade malt colonies grown for 9 weeks yielded the largest $\Delta \mathrm{E}$, it was not significantly larger than all other growth conditions. It was significantly different compared to all PDB (potato dextrose broth) conditions $(p<0.0001)$ and brewer's malt conditions $(p<0.0001)$, other than woodchip amended plates grown for 7 and 9 weeks. Shaking lab grade malt cultures at week 9 were also significantly different in comparison to all honey growth conditions, apart from week 9 woodchip-amended plates $(p=1)$ and static honey cultures $(p=1)$. Lab-grade malt shaking cultures showed the highest $\Delta \mathrm{E}$ value in week 3 and were significantly different from all other conditions in that week $(p>0.07)$.

While overall xylindein production in liquid cultures was comparable to production in woodchip plates, higher levels of pigment were produced in liquid cultures in earlier growth stages. The growth rate in shaking lab grade malt cultures was sufficient to meet the concentration of standard xylindein solution $\left(\Delta \mathrm{E}=\mathrm{L}^{*}=82.28( \pm 2.0), \mathrm{a}^{*}=-11.06( \pm 2.0)\right.$, and $\left.\mathrm{b}^{*}=-5.40( \pm 2.0)\right)$ used in previous publications $[7,46]$ after only 5 weeks of incubation. In week 5 , color values were $L^{*}=83.92, a^{*}$ $=-9.83, b^{*}=-6.20$ for shaking cultures, equal to the standard, and by week 9 these values had exceeded the standard, reaching the considerably darker $L^{*}=77.64, a^{*}=-7.61, b^{*}=-5.178$. As the standard was based upon mean values of cultures grown for 2-3 months and then triple-extracted [7], this growth method showing higher concentrations in a fraction of the time is considerably faster. In addition, extracted pigment from the same strain in malt shaking cultures at week $5(\Delta \mathrm{E}=21.1 \pm 10.8)$ was roughly equivalent to previous work where $\Delta \mathrm{E}$ of $\approx 17$ [24].

Chlorociboria colony sizes seen were also comparable to those reported in previous literature. Robinson et al. [17] achieved full plate colonization of Chlorociboria aeruginascens in 26 days, with colony diameters limited by plate size of $17.6 \pm 7.7 \mathrm{~mm}$. This was equivalent to the maximum colony diameter seen in Robinson and Laks, which was obtained in 4 weeks but persisted over a period of 4 months [47]. Tudor et al. [22], using the same methodology and strain, had cultures over twice that size, which may be ascribed to differences in species or strain tested. This decrease in speed of growth may be due to the effects of laboratory storage, which has been seen to affect growth rate [17]. The increase in colony diameter shown in these results upon addition of woodchips also mirrors previous results $[17,22]$, although it was only significant for three varieties of media after 9 weeks of growth. Finally, Stange et al. [25] reported larger colonies after a period of 10 weeks of growth; 
however, they did not begin to pigment until late in their growth, and a later paper focusing on pigmentation reported a colony radius of $\approx 22 \mathrm{~mm}$ after 8 weeks [48].

While comparisons of colony diameter have been used in most historical work and are therefore used as a point of comparison, pigment production was found to be only inconsistently related to colony size. Week 9 woodchip lab-grade malt cultures were not significantly different in terms of colony radius in comparison with brewer's malt at weeks 7 and $9(p=0.99,1)$, or honey at weeks 5 , 7 , and $9(p=0.54,0.48,1.0)$. Extracted pigment from the lab-grade malt plates at week 9 was also not significantly different $(p=1)$ from brewer's malt at week 7 and $9(p=1)$, but was only equivalent to honey at week $9(p=1)$, and also lab-grade malt at week $7(p=1)$. In addition, despite a high mean colony size in honey plates at week 5 , no similar increase in pigment production was seen. This suggests surface colonization of media only. Hence, while colony diameter generally corresponds to extractable pigment in cultures, this relationship is not a reliable measure.
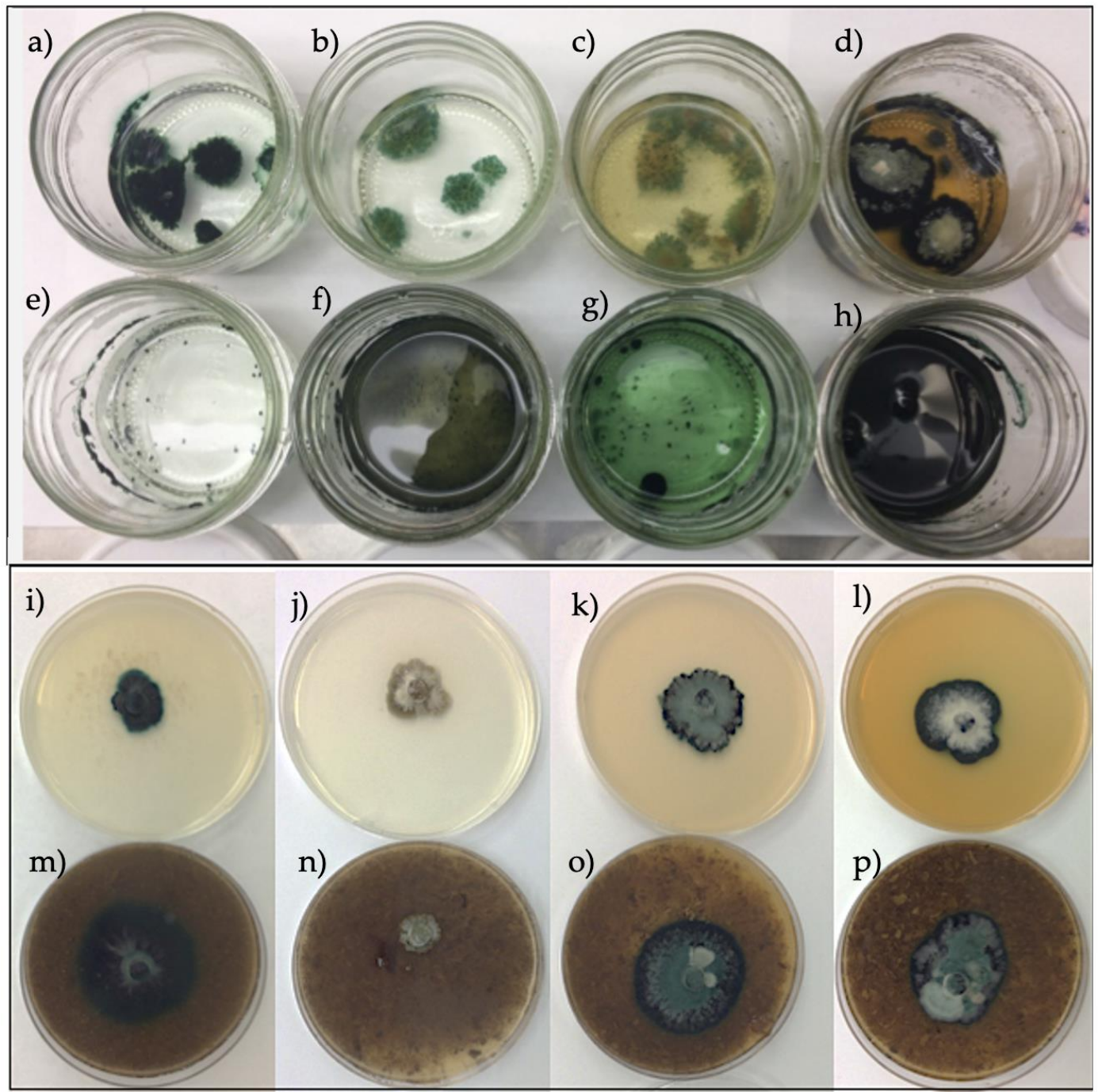

Figure 1. Cultures of Chlorociboria aeruginosa in all tested media at 9 weeks. All liquid cultures were shaken at $99 \mathrm{rpm}$. (a) Static honey liquid culture; (b) static potato dextrose broth (PDB) liquid culture; (c) static brewer's malt liquid culture; (d) static lab-grade malt liquid culture; (e) shaking honey liquid culture; (f) shaking PDB liquid culture; (g) shaking brewer's malt liquid culture; (h) shaking lab-grade malt liquid culture; (i) honey agar culture; (j) PDB agar culture; (k) brewer's malt agar culture; (1) lab-grade malt agar culture; (m) honey woodchip agar culture; (n) PDB woodchip agar culture; (o) brewer's malt woodchip agar culture; (p) lab-grade malt agar culture. 


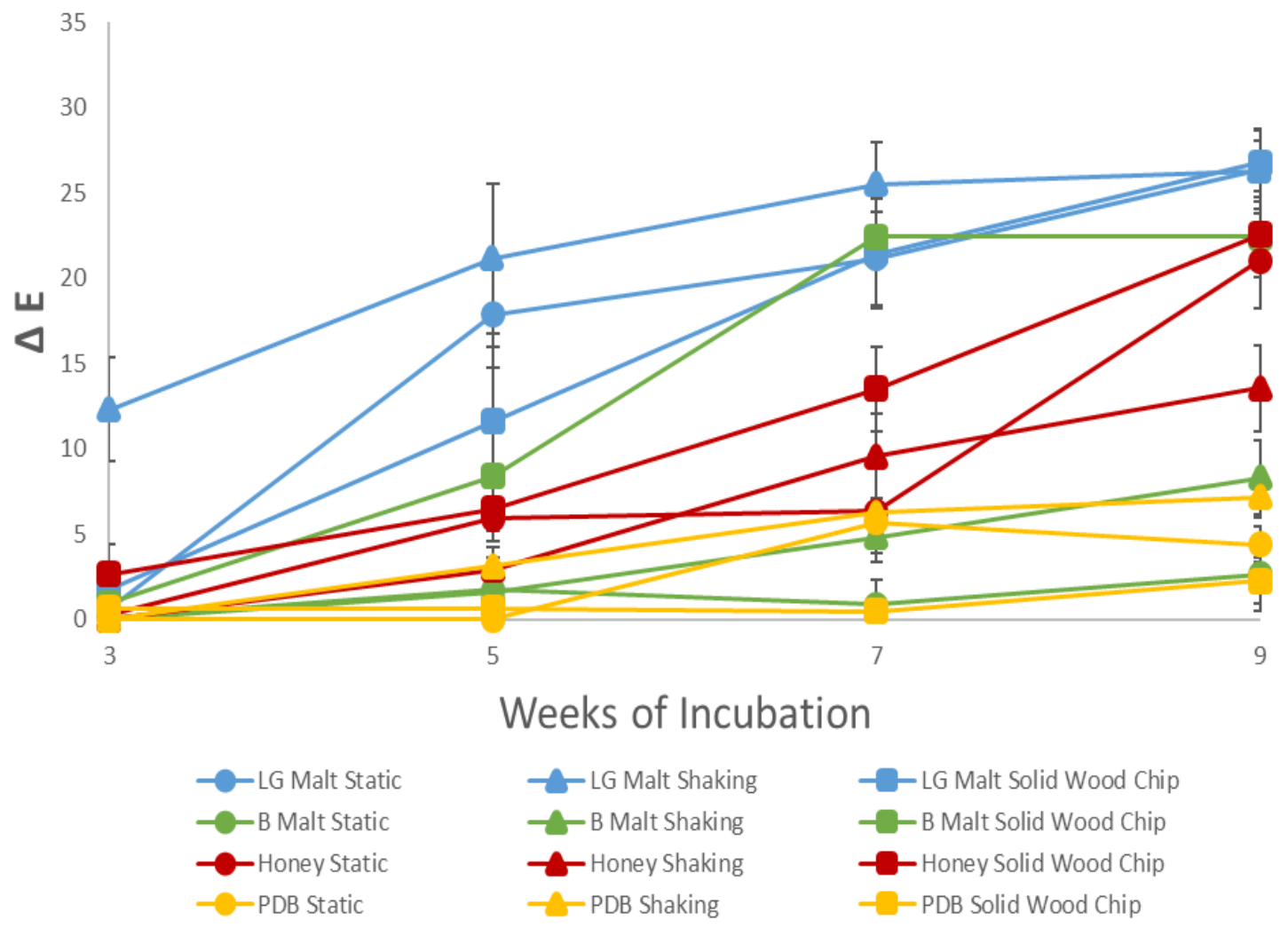

Figure 2. Mean color difference $(\Delta \mathrm{E})$ resulting from variation of media and cultivation technique. Error bars represent standard error. Interaction between media, week, and growth conditions tested with a Proc Glimmix model was found to be highly significant ( $\mathrm{df}=18,150, F$-value $=13.47, p<0.0001$ ). Lab-grade (LG) bacteriological malt cultures achieved a much higher $\Delta \mathrm{E}$ by week 3 , showing the fastest pigment production by far compared to other media at that time. Lab grade media also showed the largest $\Delta \mathrm{E}$ value by week 9 , followed by brewer's (B) malt woodchip plates and honey woodchip plates. Potato dextrose broth (PDB) showed the least pigmentation across cultivation techniques.

In addition, previous research has also shown $\mathrm{pH}$ to be a significant factor in pigmentation of Chlorociboria aeruginascens, with the highest pigmentation reported at $\mathrm{pH} 2.5$ and 5 in malt agar plates [49] and at $\mathrm{pH}$ of 4 in orange juice medium [48]. Here, the $\mathrm{pH}$ of liquid cultures was tested before inoculation and at the end of nine weeks of growth. Overall, media became less acidic after fungal growth (Table 1). Pigment formation in malt extract agar (MEA) at different $\mathrm{pH}$ values in this range (as tested by Tudor, Robinson and Cooper [49]) showed brownish pigmentation at a $\mathrm{pH}$ of 7, and dark green pigmentation at $\mathrm{pH}$ values of 5 and 6 . Overall, this aligns well with morphology seen in malt media. However, the lack of pigmentation seen in pH 5 PDB, and the dark pigmentation in honey, show that the differences were not due to $\mathrm{pH}$ changes but down to the differences in growth conditions.

Table 1. $\mathrm{pH}$ of liquid media before and after Chlorociboria growth.

\begin{tabular}{ccccc}
\hline Growth Condition & Honey & Brewer's Malt & Lab-Grade Malt & PDB \\
\hline Pre-growth & 6 & 6 & 6 & 5 \\
Static post-growth & 7 & 6 & 5 & 5 \\
Shaking post-growth & 7 & 6 & 5 & 6 \\
\hline
\end{tabular}

The superior performance of shaking liquid cultures may have been due to multiple factors. As an aerobic organism, increased oxygen availability in liquid culture may have facilitated higher levels of growth. While the volumetric oxygen transfer coefficient $(\mathrm{kLa})$ was not determined, agitation 
of cultures is standard in bioreactors to facilitate increased gas exchange, and both agitation and oxygenation have been found to influence secondary metabolite production in filamentous fungi [50,51]. However this explanation may not fully explain the results, as previous work has found that increased level of oxygen was not associated with increases in pigment production [48]. In addition to the possible influence of increased oxygen availability, the shear stresses experienced by the fungi in shaking liquid cultivation may have led to breakdown of fungal hyphae. This could have led to increased pigmentation as a stress response.

The superior pigmentation of Chlorociboria aeruginosa grown on malt extract confirmed results from other researchers $[19,20]$. It was hoped that there would not be a significant difference between the bacteriological malt extract and the more economical brewer's malt extract, allowing for cheaper pigment production. However, while brewer's malt performed well in woodchip plates, it did not compare to the pigment production from shaking lab-grade malt cultures. Overall, ratios of four tested sugars were similar between malt varieties, with bacteriological-grade malt containing a slightly higher percent maltose $(76.93 \%$ vs. $73.35 \%)$. As maltose has been identified as a preferred carbon source, this may help account for color discrepancies, although this may also be down to nitrogen availability, which has been suggested to have more of an effect on pigmentation of Chlorociboria species [21]. The minimal pigmentation seen in the PDB cultures' solid culture was also reflective of previous research, with potato starch described as producing brown and light green coloration of colonies in solid growth substrate [20,22].

Honey resulted in good growth and pigmentation, equivalent to or better than all other growth medias and conditions. The honey mostly contained glucose and fructose, with smaller amounts of maltose and sucrose. The pigmentation produced by this combination is consistent with studies showing pigmented Chlorociboria growth in fruit juices $[20,21,25,26,48]$, which normally contain glucose, fructose, and sucrose. Glucose media in particular have been described leading to pigmented growth [19-21].

Variations of growth morphology are more likely indicative of varying levels of nitrogen present in media, and fungal stress responses. While nitrogen content was not analyzed in these tested media, other work on media where nitrogen is not a limiting factor has shown fungal metabolism, focusing on biomass production with pale growth until late in colony life when available nitrogen has likely been depleted [21]. Determination of sugar concentration and biomass were also not assessed due to the blending and extraction methodology interfering with measurements. Biomass in particular was not feasible due to the very fine texture of fungal particles and their tendency to stick to separatory funnel walls. Other work [21] has shown that pigment production in Chlorociboria does not directly relate to biomass, and the pattern of high biomass not being associated with high pigmentation was seen in the preliminary media screen, where multiple media only started to show pigmentation after months of colony growth, and in pale growth in early weeks for all media apart from honey. Honey showed high levels of pigmentation and visibly low levels of biomass growth, supporting a metabolic switch from growth to pigmentation. However, this switch was also seen to be associated with other growth conditions, as shown in PDA plates, which began to pigment only on addition of woodchips. As xylindein production has been seen to occur in response to environmental stressors [48], it is likely that these woodchips provided the stress that induced pigmentation, perhaps through containing secondary metabolites of other decay fungi once present in the wood.

\subsection{Pigment Variation across Chlorociboria Species and Strains}

Xylindein production was seen to vary over time, between strains, with a significant difference seen for the interaction between growth condition (liquid media vs. woodchip), week, and fungal strain $((\mathrm{df}=27,144), F=47.93, p<0.0001)$. The highest mean color difference $(\Delta \mathrm{E})$ of $26.45( \pm 0.44)$ was seen in extracted pigment from $C$. aeruginascens UAMH 11655 grown on woodchip-amended plates for 9 weeks (Figure 1). However, while significantly different from all values in weeks 1 through 4 , and 
all control values $(p<0.0001)$, it did not significantly differ after week 5 (aside from C. aeruginascens UAMH 7614 woodchip plates at week $5(p<0.0001))$.

While cultures resulted in similar levels of extracted pigment in later weeks, early weeks showed different pigment production patterns. By week 2, $\Delta \mathrm{E}$ of $C$. aeruginosa UAMH 11657 was significantly different than the liquid media control $(p<0.0001)$. C. aeruginosa UAMH 11657 also showed a significant difference from $C$. aeruginascens UAMH $7614(p<0.0001)$. In weeks 3 and 4 , liquid cultures generally performed better than woodchip plate cultures, with C. aeruginascens UAMH 7614 yielding the highest mean color changes (Figure 3). In week 3, C. aeruginascens UAMH 7614 in liquid media showed a significantly higher value than in its pigment production in wood $(p<0.0001)$ or compared to woodchip cultures of $C$. aeruginosa UAMH 11657 and control $(p=0.001)$. In week $4, C$. aeruginascens UAMH 7614 still had the highest mean of $20.76( \pm 1.00)$, and showed a significant difference compared to all other tested conditions apart from C. aeruginosa UAMH 11657 culture $(p=0.3764$ liquid media, $p=0.51$ solid media).
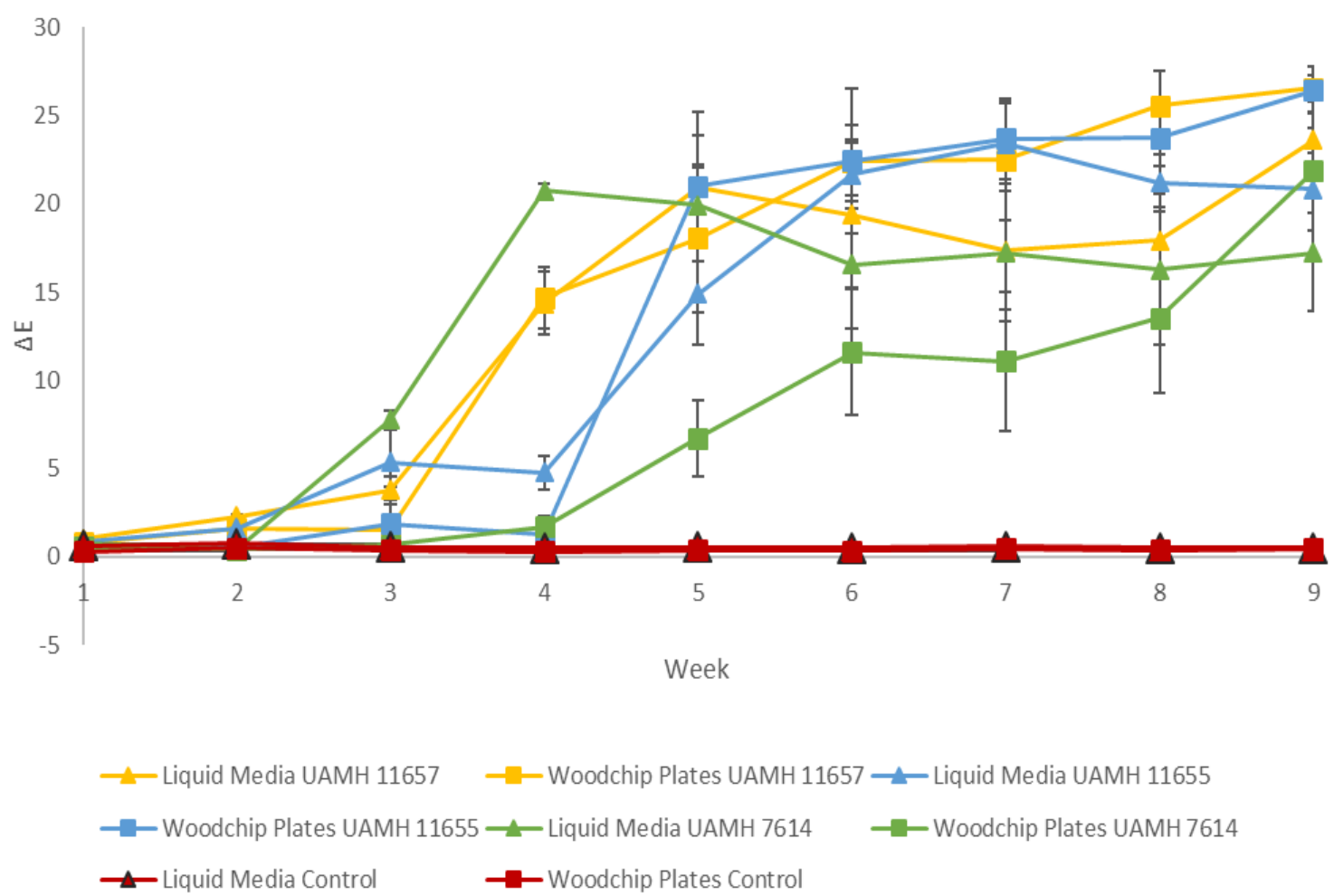

Figure 3. Differences in color $(\Delta \mathrm{E})$ produced by Chlorociboria sp. over 9 weeks growth. Strain UAMH 11657 was Chlorociboria aeruginosa, and strains UAMH 11655 and UAMH 7614 were Chlorociboria aeruginascens. Error bars represent one standard error. A significant difference was seen for the interaction between growth substrate (liquid media vs. woodchip), week, and fungal strain ( $\mathrm{df}=27$, 144), $F=47.93, p<0.0001)$. No significant differences were seen after week 5 in $\Delta \mathrm{E}$ between fungal cultures.

Cultures showed consistent morphology when grown in liquid culture, with color variation (Figure 4). Most notably C. aeruginascens UAMH 11655 by week 9 had media that appeared somewhat yellow, whereas the other cultures were more consistently blue/green. Upon blending, these differences were no longer as apparent. These visual differences indicate variability in secondary metabolite production between cultures, and inhomogeneity of extractions. 

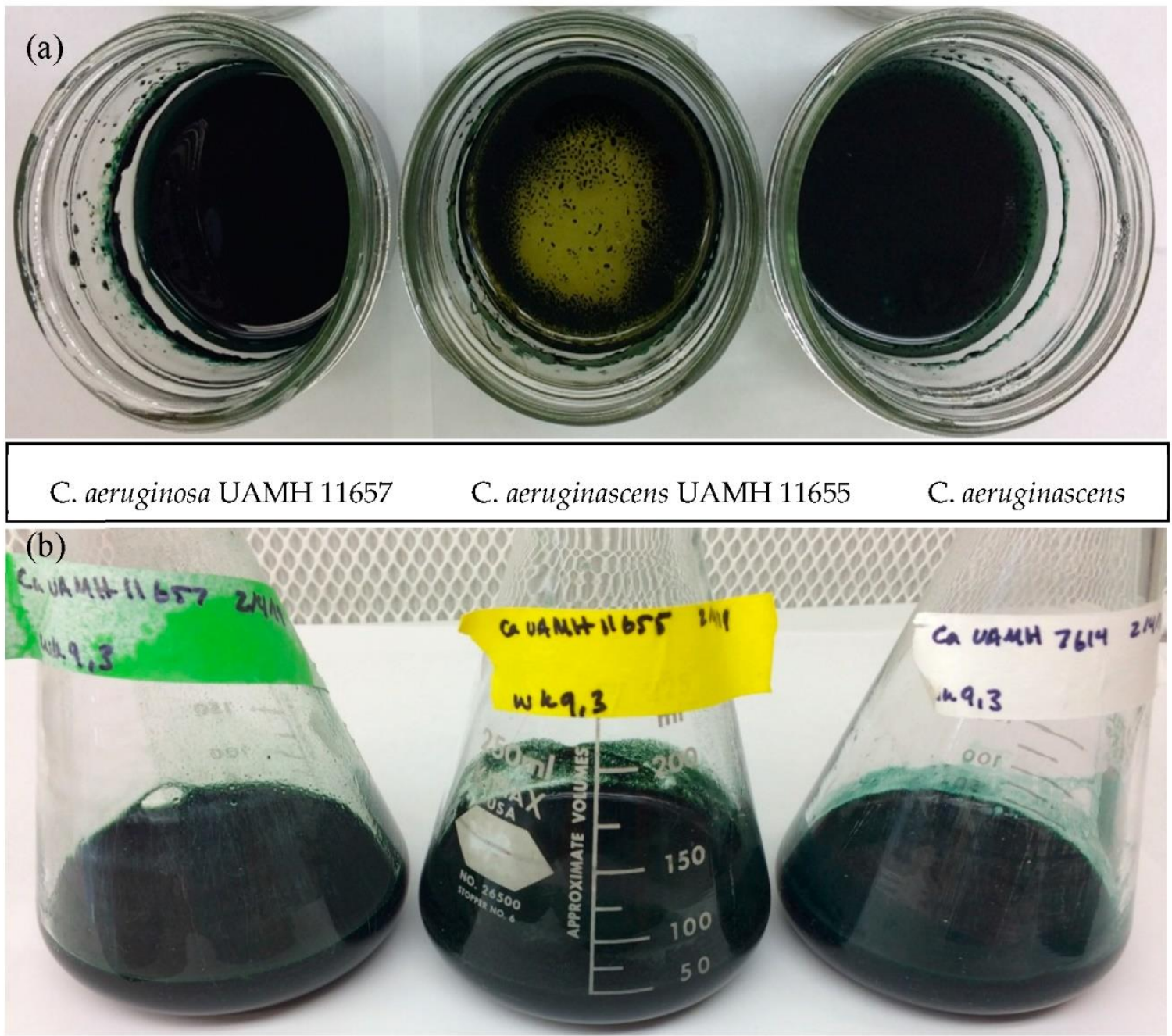

Figure 4. Liquid cultures of tested Chlorociboria species and strains in lab-grade malt extract liquid media at 9 weeks of growth. (a) Shaking cultures; (b) the same shaking cultures after blending 3 min to break up hyphae.

Extraction results mirrored colony radius on amended woodchip plates (Figure 5), as was seen in comparisons of growth conditions. A significant difference was seen between species radii over time (df $(16,54), F=4.34, p<0.001)$ ). However, on the basis of Tukey comparisons, we found no significance within each week between strains until week 8; there was a significant difference between C. aeruginascens UAMH 7614 and $C$. aeruginascens UAMH $11655(p=0.0037)$, where strain UAMH 11655 had the highest mean radius overall with a value of 19.5 ( \pm 4.3$)$. C. aeruginosa UAMH 11657 was found to have the highest mean size in week 9 , although this was not significantly different from week 5 ( $p=0.25$ ), or from $C$. aeruginascens UAMH 11655 and $C$. aeruginosa UAMH 11657 in any of the following weeks $(p>0.1)$. Week 9 also showed variations in colony morphology (Figure 6), with C. aeruginascens UAMH 7614 lacking a dark border around the colony edge, which was seen in the other two strains. 


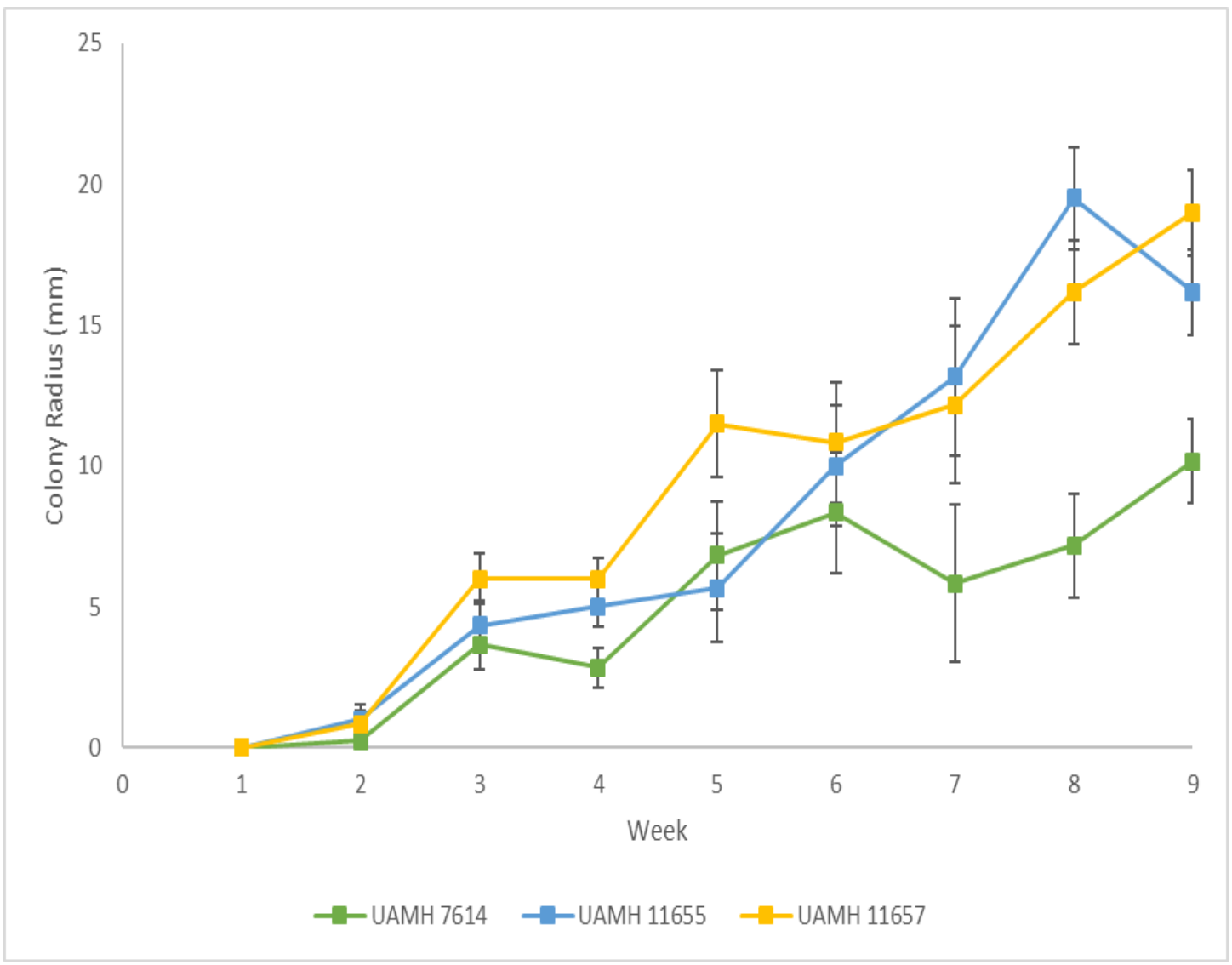

Figure 5. Comparison of colony radius and Chlorociboria variety in lab-grade malt agar media. UAMH 7614 and UAMH 11655 were both C. aeruginascens, with UAMH 11657 C. aeruginosa. Errors bars represent one standard error. A significant difference was seen over time (df $(16,54), F=4.34, p<0.001)$.
C. aeruginosa UAMH 11657
C. aeruginascens UAMH 11655
C. aeruginascens UAMH 7614

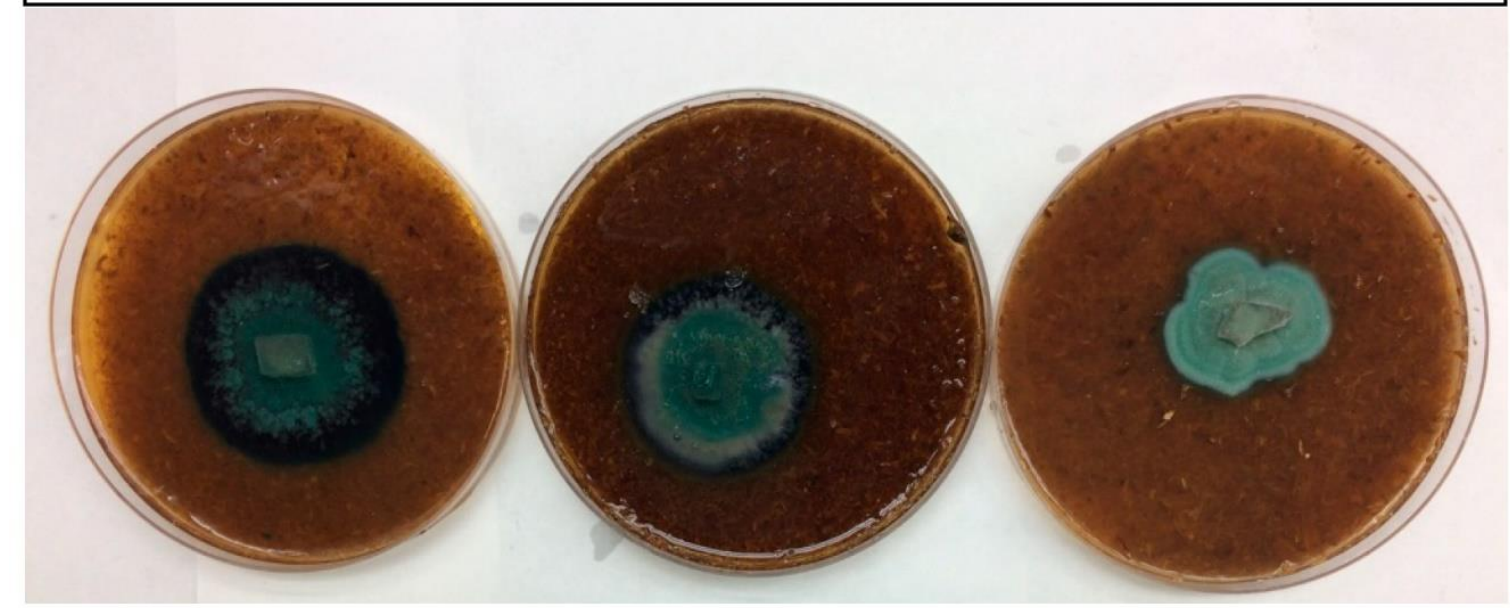

Figure 6. Woodchip-amended lab-grade malt media plate cultures of species and strains at 9 weeks of growth.

These results confirm previous research showing variation in colony size between pigmenting species and strains of Chlorociboria [17,22]. In addition, it lends support to the assumption that larger 
colony sizes between different species and strains are associated with increases in actual extractible pigment. This was demonstrated by C. aeruginascens UAMH 7614, which showed a consistently smaller colony diameter in comparison with the other species/strains tested, and showed lower mean $\Delta \mathrm{E}$ values in woodchip plates also, although this difference was not significant. As it has been hypothesized that pigment production redirects metabolic energy away from biomass production [21], this result was important to confirm.

Comparisons of extracted pigment (color only) were similar to what was seen by Weber et al. [22], who compared only one strain of $C$. aeruginascens and C. aeruginosa in liquid cultures alone. In that study, C. aeruginosa UAMH 11657 produced a $\Delta \mathrm{E}$ of $\approx 17$ after 6 weeks and 6 days (48 total days), compared to $C$. aeruginascens UAMH 7615, which showed little increase in color change after 4 weeks (final $\Delta \mathrm{E}$ of $\approx 12$ ) [24]. In comparison, in this study liquid cultures at 7 weeks (C. aeruginosa strain $\mathrm{UAMH} 11657)$ had a comparable $\Delta \mathrm{E}$ value of $17.36 \pm 7.3$. However, $C$. aeruginascens cultures performed equally well, instead of exhibiting plateauing pigment production (UAMH 7614 with mean of $17.24 \pm$ 8.13 and UAMH 11655 of $23.42 \pm 5.04$ ). While no significant differences were seen between species or strains, 5 weeks was the cut off above which no significant differences were seen between radius size and final colony radius for C. aeruginosa UAMH 11657 and C. aeruginascens UAMH 11655. It is notable that Weber et al. [24] described pigmentation for C. aeruginascens strain 7615 tapering off after 4 weeks, as this also correlates fairly well to weeks 4 and 5 , marking a point at which differences between strains are no longer significant. However, this may also be due to limitations in the extraction method used, with pigment not able to transfer from media to DCM after a certain saturation point (lower than xylindein total saturation point).

Despite no statistically significant difference between samples after week 6 , we found that $L^{*} a^{*} b^{*}$ color values showed strain-based variation in comparison to the standard color values used for standardizing xylindein extraction. The standards were originally developed as an indirect method of quantifying pigment concentration in extract solutions on the basis of a triple extraction of a single plate of $C$. aeruginosa UAMH 11657 grown in woodchip plates for 2-3 months, in $50 \mathrm{~mL}$ of DCM [6], with solutions considered standardized if color values fell within \pm 2.0 of the values $L^{*}=82.28$, $a=-11.06$, and $b=-5.40$. It should be noted that at present there is no method of converting color value to concentration. Mathematical relationships have been established for the pigment produced by Scytalidium cuboideum, anther spalting fungi [52]. Use of color values as a measure of concentration has been validated [24]; however, limited amounts of xylindein and a lack of purification techniques have prevented modeling this relationship thus far.

Using the above as a measurement bar, average values of $C$. aeruginosa UAMH 11657 woodchip plate cultures at week 7 were the only test condition that "met" this standard (Table 2). C. aeruginosa UAMH 11657 woodchip cultures (weeks 8 and 9) showed lower $L^{*}$ values (the extract was darker, which indicates a higher concentration of xylindein) but otherwise fell within the standard range. However, on the basis of average values alone, several other cultures showed variation in the $a^{*}$ value, while $L^{*}$ and $\mathrm{b}^{*}$ values were within range, resulting in "greener" colors (Table 2 ). These included C. aeruginosa UAMH 11657 in liquid media by week 9 and C. aeruginascens UAMH 11655 in all conditions. Woodchip C. aeruginascens UAMH 11655 were also slightly out of range in terms of $b^{*}$ values. However, range of samples shown in standard deviation values showed that even though average values did not fall within the range of the sample, this difference may not be supported statistically. As fungi are biological organisms, variation and inhomogeneity in extracts from them is expected. However, the fact that so many values appear "greener" suggests that there is variation in pigment production between samples.

Using the lab standard color chart and previous literature on Chlorociboria color variation, it is possible to hypothesize about color variance in xylindein and other secondary metabolites. The "greener" color likely indicates production of additional pigmented secondary metabolites occurring at different rates, resulting in differences in overall culture coloration. $L^{*}$ values, representing the darkening of solution from increased pigmentation, have been noted to be the most associated with fungal pigment 
concentration in previous work [52]. However, variation of culture color is well known in Chlorociboria sp. Culture colors ranging from blue-green to lime were reported by Frenzel [20], implying the presence of a yellow compound, and Edwards and Kale [53] reported a yellow compound they identified as a xylindein quinol. A yellow pigment was also noted by Weber et al. [24], the amount of which decreased with colony age, and it was suggested to be a potential breakdown or precursor of xylindein. Variation in production of this yellow compound between species may be responsible for the color values with more negative $a^{*}$ values. The difference is not seen in the $b^{*}$ values, likely because $b^{*}$ values range from yellow to blue, and presence of a yellow compound may be outweighed by the bluer xylindein, resulting in no overall changes on this scale.

Table 2. Chlorociboria extraction colors with $\mathrm{L}^{*}$ values matching or below standard. Extractions within the lab standard are denoted with an asterisk, and values out of range are in bold. Average color values of $a^{*}$ were seen to go out of range of standard, while $L^{*}$ and $b^{*}$ values conformed, apart from C. aeruginascens UAMH 11655 woodchip plate samples.

\begin{tabular}{|c|c|c|c|c|c|}
\hline Chlorociboria Source & Week & $\mathrm{L}^{*}$ & $a^{*}$ & $\mathbf{b}^{*}$ & Color \\
\hline Standard (Hinsch 2015) * & $8-12$ & $82.28( \pm 2.00)$ & $-11.06( \pm 2.00)$ & $-5.40( \pm 2.00)$ & \\
\hline $\begin{array}{l}\text { C. aeruginosa UAMH } 11657 \\
\text { woodchip plate * }\end{array}$ & 7 & $82.83( \pm 7.18)$ & $-10.43( \pm 3.08)$ & $-6.68( \pm 1.40)$ & \\
\hline $\begin{array}{l}\text { C. aeruginosa UAMH } 11657 \\
\text { woodchip plate }\end{array}$ & 8 & $77.36( \pm 1.37)$ & $-7.68( \pm 2.62)$ & $-6.47( \pm 1.04)$ & \\
\hline $\begin{array}{l}\text { C. aeruginosa UAMH } 11657 \\
\text { woodchip plate }\end{array}$ & 9 & $75.96( \pm 1.61)$ & $-6.96( \pm 2.04)$ & $-6.17( \pm 0.75)$ & \\
\hline $\begin{array}{l}\text { C. aeruginosa UAMH } 11657 \text { liquid } \\
\text { media }\end{array}$ & 9 & $83.05( \pm 4.45)$ & $-13.32( \pm 3.20)$ & $-6.62( \pm 2.09)$ & \\
\hline $\begin{array}{l}\text { C. aeruginascens UAMH } 11655 \\
\text { woodchip plate }\end{array}$ & 7 & $83.97( \pm 6.40)$ & $-13.47( \pm 4.70)$ & $-7.65( \pm 0.76)$ & \\
\hline $\begin{array}{l}\text { C. aeruginascens UAMH } 11655 \\
\text { woodchip plate }\end{array}$ & 8 & $83.62( \pm 5.22)$ & $-13.74( \pm 3.87)$ & $-7.44( \pm 0.04)$ & \\
\hline $\begin{array}{l}\text { C. aeruginascens UAMH } 11655 \\
\text { liquid media }\end{array}$ & 7 & $83.06( \pm 6.74)$ & $-13.28( \pm 0.05)$ & $6.67( \pm 0.89)$ & \\
\hline
\end{tabular}

The variation in colors seen between strains suggests that using standard color values, as has been done in multiple publications $[3,7,9,54]$, may not be appropriate for use on pigment extracted from cultures that are not $C$. aeruginosa UAMH 11657 grown on woodchips, or at the very least, the $\mathrm{a}^{*}$ values should have a wider range of acceptability for research or applications where any coloration produced by Chlorociboria sp. is acceptable. Indirect measures of concentration should be based primarily on $L^{*}$ values similar to $S$. cuboideum [52], and specific strains and growth conditions should be used if achieving a specific color is desired.

\subsection{Comparison of Extracted Xylindein Purity and Electronic Activity}

In order to assess purity of xylindein produced by different strains/species and across growth conditions, we compared absorption spectra. For comparisons of $C$. aeruginosa across growth conditions, we took spectra on week 9 xylindein extractions (Figure 7), with the exception of PDB cultures that produced so little xylindein they could not be tested effectively. Xylindein shows a dominant peak 
at the $\approx 660 \mathrm{~nm}$ wavelength region in DCM, with $S_{0}-S_{1}$ absorption in the $550-700 \mathrm{~nm}$ range $[10,12]$, which could be seen in all samples. Purity of samples was determined on the basis of absorption in the $400 \mathrm{~nm}$ region, where contaminants in xylindein solutions have been previously described as causing increased absorption [12]. Comparisons showed that lab-grade (bacteriological) malt cultures grown in shaking conditions showed the lowest absorption around $400 \mathrm{~nm}$, appearing quite close to control ethanol-washed xylindein samples [26]. Brewer's malt cultures in general showed high contaminant levels as reflected in high absorption around this area, with honey cultures showing reduced contaminants in comparison. It is notable that cultures associated with the highest pigmentation levels (lab-grade malt and honey cultures) showed lower overall levels of absorption at $400 \mathrm{~nm}$. Finally, woodchip cultures generally showed higher absorption around $400 \mathrm{~nm}$, in comparison to liquid cultures of the same media, likely due to extractives from the wood.
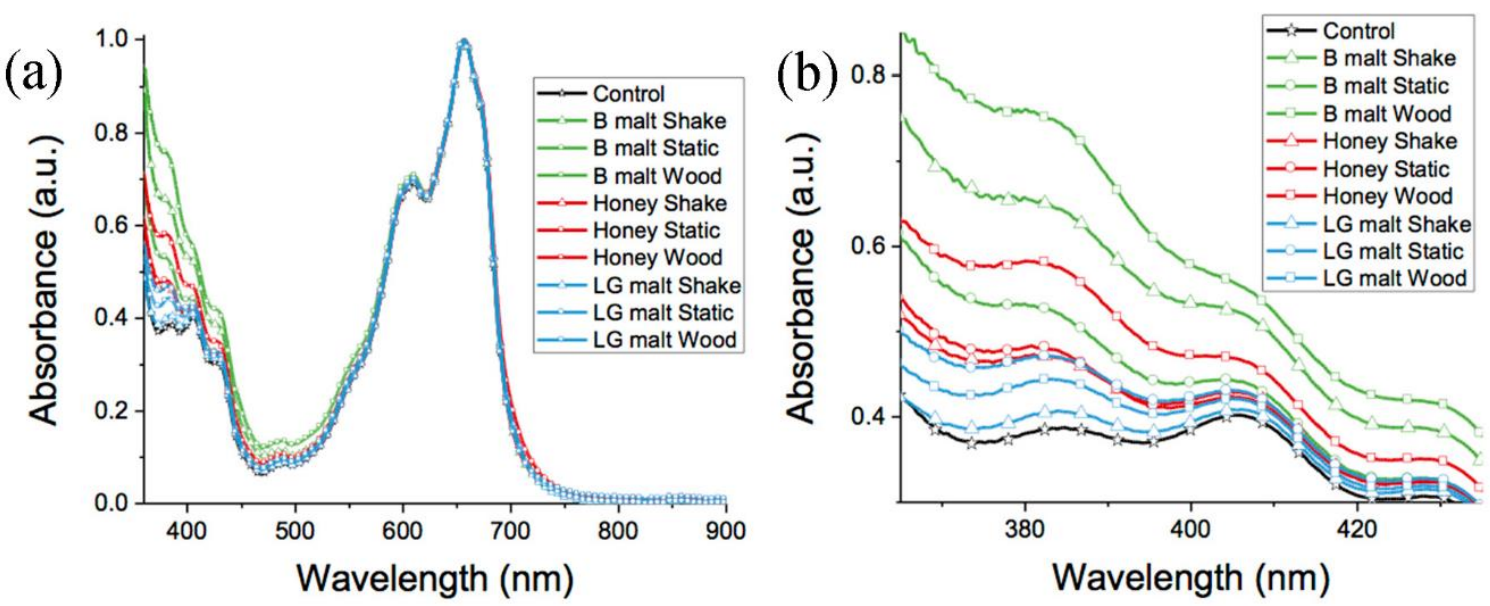

Figure 7. Xylindein extract from C. aeruginosa (UAMH 11657) absorption across growth conditions. Control sample was ethanol-washed solidified xylindein. (a) Absorbance of extracted pigment solutions showing characteristic xylindein peak at $\approx 660 \mathrm{~nm}$ and variation in absorbance at shorter wavelengths due to varying concentration of non-xylindein interferents known to reduce electronic properties. (b) Close up absorbance around $400 \mathrm{~nm}$, where largest variation was seen in terms of other interfering compounds in solution. Higher absorbance is associated with higher concentrations of contaminants, with lab-grade shaking cultures showing lowest contamination.

Optical data provided limited information on variation in production of secondary metabolites for comparisons of species and strain, and therefore xylindein quality (Figure 8). Xylindein's dominant peak was seen in the $\approx 660 \mathrm{~nm}$ wavelength region in DCM, with $S_{0}-S_{1}$ absorption in the 550-700 nm range [13,14]; however, the differences in the absorption at around $400 \mathrm{~nm}$ was relatively small. Therefore, no determinations of purity differences between strains were made on the basis of these data.

Conductivity of xylindein extract from tested species and strains were compared, and differences in conductivity were seen in samples both before and after an ethanol wash (Figure 9), with the post-wash C. aeruginascens UAMH 7614 showing the highest electric currents in the current-voltage (I-V) characteristics in produced thin films. It is notable that these clear differences between strains were not seen in optical data, indicating other interfering compounds are likely present in samples that were not contributing to the enhanced absorption in the $370-450 \mathrm{~nm}$ range previously described. C. aeruginascens UAMH 11656 showed less improvement when subjected to an ethanol wash compared to $C$. aeruginosa UAMH 11657, which may indicate increased production of secondary metabolites not soluble in ethanol. Finally, effective electron mobilites were calculated using fits of the post-wash data (Figure 10) to Equation (2) (see Materials and Methods), with C. aeruginascens strain UAMH 7614 showing the best effective electron mobility at $0.012 \mathrm{~cm}^{2} /(\mathrm{Vs})$, followed by $C$. aeruginosa strain UAMH 11657 at $0.0035 \mathrm{~cm}^{2} /(\mathrm{Vs})$ and finally C. aeruginascens strain UAMH 11655 at $8.2 \times 10^{-4} \mathrm{~cm}^{2} /(\mathrm{Vs})$. 
The highest effective mobility of $0.012 \mathrm{~cm}^{2} /(\mathrm{Vs})$ is comparable to $0.01 \mathrm{~cm}^{2} /(\mathrm{Vs})$ previously obtained in post-wash wild-type xylindein films in the same device geometry [15]. However, these values are considered to be a lower limit to the intrinsic mobility, as considerably higher values of up to $0.4 \mathrm{~cm}^{2} /(\mathrm{Vs})$ were reported in similar films in a more optimized device geometry [15].

While C. aeruginascens UAMH 7614 showed the slowest pigment production and smallest colony sizes, it was also associated with highest quality xylindein, as shown in (I-V) characteristics from xylindein films. This may be due to larger allocation of resources away from growth and towards production of pigment, a pattern that has been suggested elsewhere [21]. It is notable that medias seen to produce higher levels of pigmentation, lab-grade malt, and honey were also associated with fewer contaminants absorbing in the $40 \mathrm{~m}$ range. This suggests that these contaminants are primarily fungal secondary metabolites associated with growth or biomass production, production of which is likely reduced with higher energy allocation to pigment production. Decreased biomass production by C. aeruginascens UAMH 7614 in early weeks could have resulted in reduced pigmentation overall; however, the pigment produced appears to have been less contaminated with other products of fungal metabolism. It is interesting to note that this strain had a different morphology of growth than the other two, lacking a deeply pigmented outer zone.

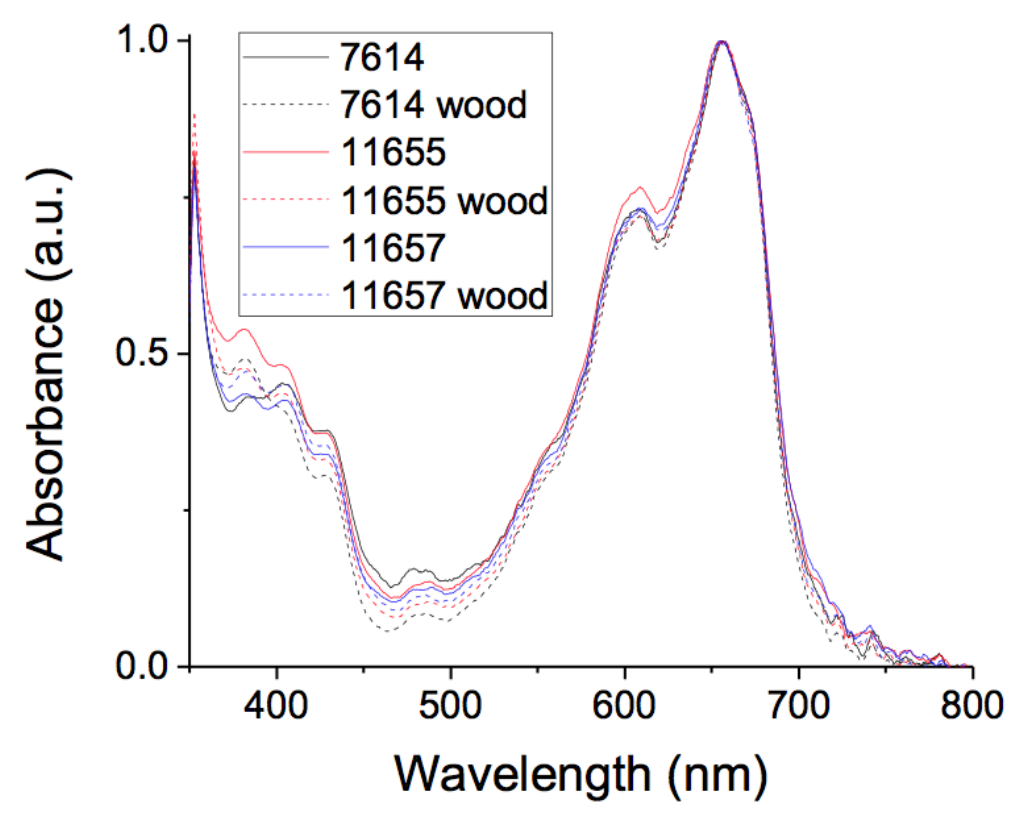

Figure 8. Xylindein absorption across growth strains and growth conditions for week 9 samples. Chlorociboria cultures (C. aeruginosa strain UAMH 11657 and C. aeruginascens strains UAMH 11655 and UAMH 7614) grown in lab-grade malt media, in either liquid culture or woodchip-amended agar plates (denoted "wood" in legend). Absorbance of extracted pigment solutions showed a characteristic xylindein peak at $\approx 660 \mathrm{~nm}$ and variation in absorbance at shorter wavelengths. Averages are given for each strain; however, a large amount of variability was seen between samples of each strain around the $400 \mathrm{~nm}$ mark. Therefore, no strain could be clearly identified as producing highest quality pigment using optical data.

In addition, the variation in values of $a^{*}$ despite $L^{*}$ and $b^{*}$ values in relation to the standard suggests that other secondary metabolites, including the yellow secondary metabolite, are being produced differentially among strains, species, and in response to different growing conditions. This is further supported by conductivity data, both before ethanol wash and in the different levels of improvement seen in response to ethanol washing. While data suggest that there is no significant difference between species in terms of extracted pigment production, differences in strain may have an effect on speed of xylindein production, especially in earlier weeks of growth. This suggests 
that stimulatory mechanisms for pigment production pathways are highly conserved in the genus, in addition to pigment production itself. Although the use of only three strains presents a somewhat limited picture, with the two species represented not being closely related in terms of phylogeny, it may be the case that most species in the genus Chlorociboria produce pigment at similar rates and in response to similar stimuli.

While this research identified growth conditions leading to amounts of xylindein produced in only 5 weeks, equivalent to $2-3$ months using previous methods, it also indicated that extraction methodology needs to be improved to allow for efficient xylindein collection. The statistical equivalence of multiple media and culture conditions by week 9 for comparisons of growth, and after week 5 for comparisons of strain indicate that there is a limit to pigment extractable into DCM using the current methodology. By week 9, liquid cultures appeared almost black due to pigment concentration, with no visible difference in color after extraction. Significant amounts of pigment produced by the fungi were therefore not captured using this extraction methodology. While other publications have used multiple extractions and combined the results to attempt to extract more of the pigment from the growth substrate [7], this process is laborious. Development of new processes, including experimentation with new solvents, is important to efficiently collect produced pigment. DCM has been the best solvent thus far [23], while other solvents have been identified to test using Hansen solubility parameters, including naphthalene, butyl acetate, and trichloroethylene [23]. Investigation into use of these and other solvents may improve efficiency of extraction, in addition to procedural changes.

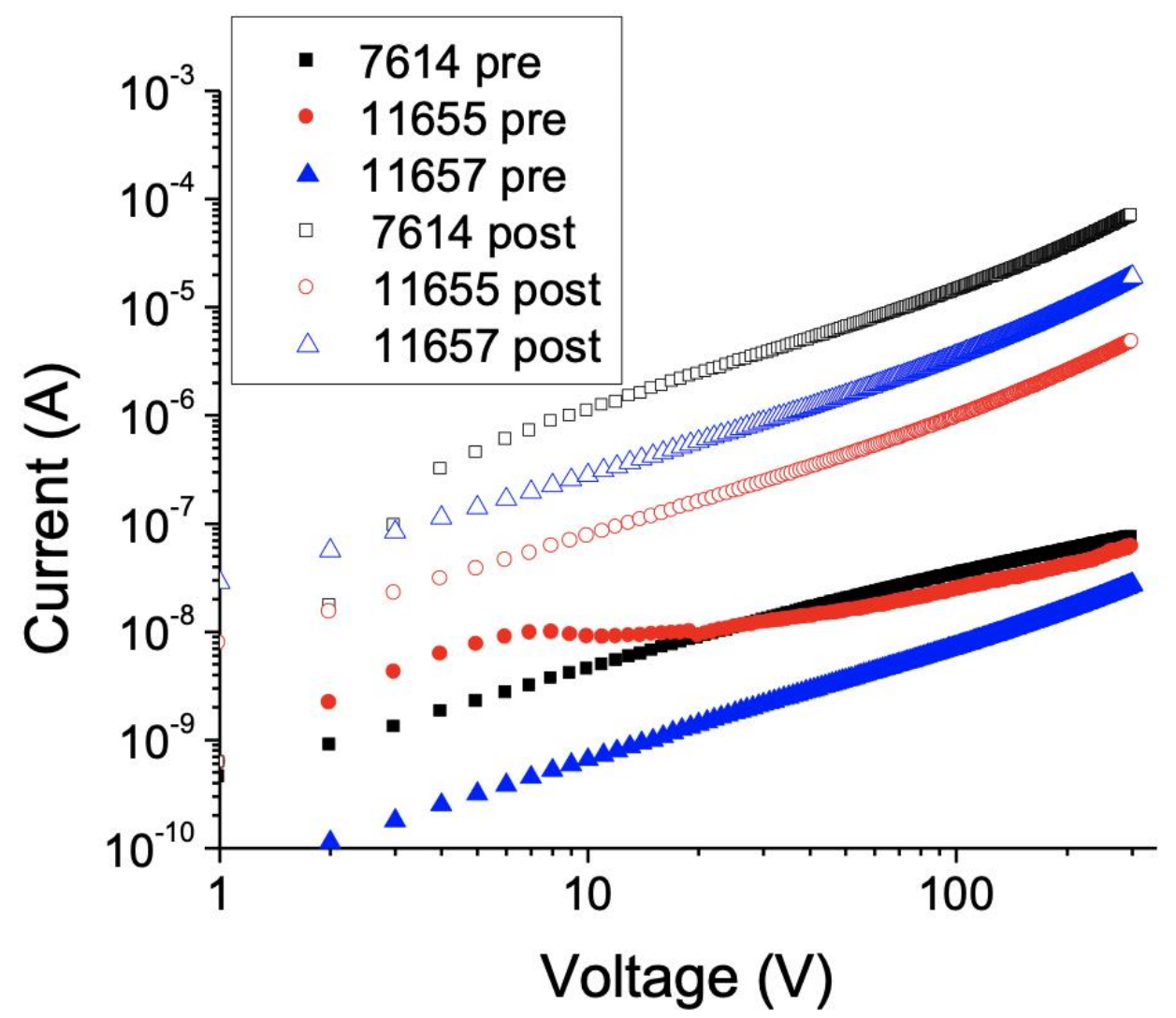

Figure 9. Current-voltage (I-V) characteristics for "pre-wash" and "post-wash" xylindein films made from extracts of different species and strains, including C. aeruginascens strains UAMH 7614 and UAMH 11655 and C. aeruginosa strain UAMH 11657. Testing was carried out on interdigitated Au electrodes with a $25 \mu \mathrm{m}$ gap. C. aeruginascens yielded higher quality xylindein, with strain UAMH 7614 showing the best results. 


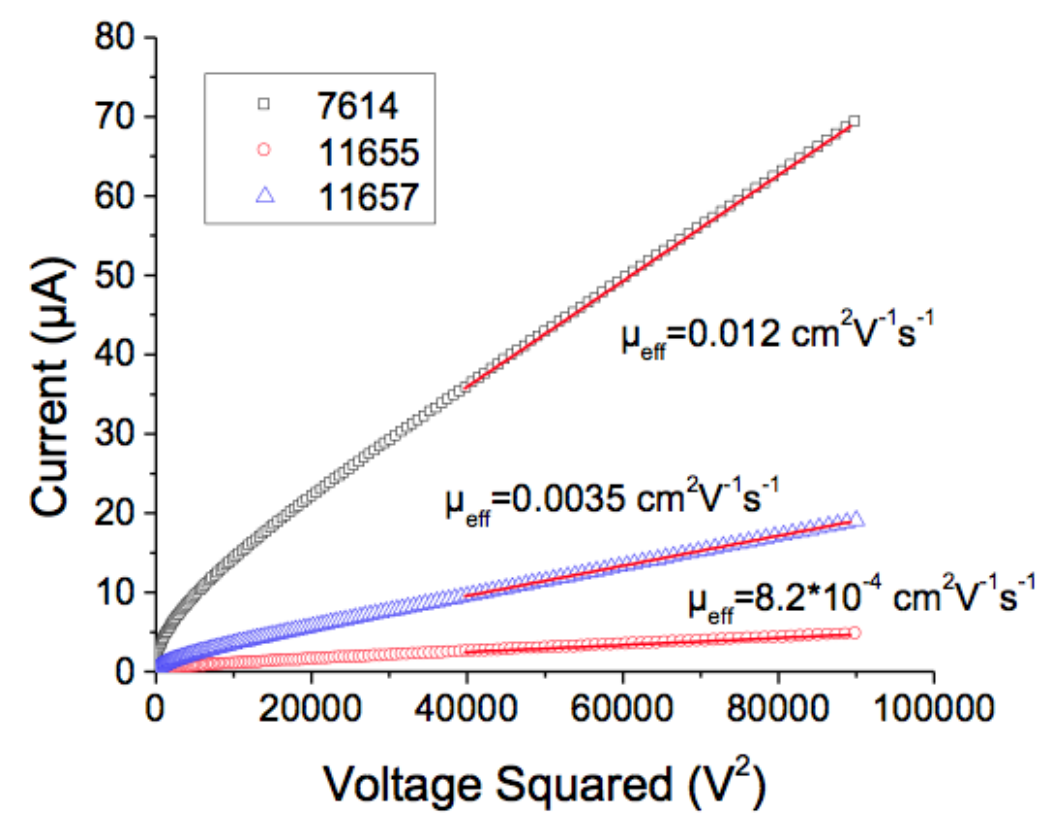

Figure 10. The current-voltage (I-V) characteristics plotted as current (I) vs. voltage squared $\left(\mathrm{V}^{2}\right)$ in xylindein films depending on species and strains, including C. aeruginascens strains UAMH 7614 and UAMH 11655 and C. aeruginosa strain UAMH 11657. Lines represent linear fits to Equation (2) from which effective mobilities were calculated. Calculations indicate that xylindein from C. aeruginascens strain UAMH 7614 showed the best effective electron mobility at $0.012 \mathrm{~cm}^{2} /(\mathrm{Vs})$, followed by C. aeruginosa strain UAMH 11657 at $0.0035 \mathrm{~cm}^{2} /(\mathrm{Vs})$ and finally C. aeruginascens strain UAMH 11655 at $8.2 \times 10^{-4} \mathrm{~cm}^{2} /(\mathrm{Vs})$.

Despite current inefficiencies in extraction methodology for liquid state growth, identification of growth conditions leading to the fastest production of quantities of xylindein has significant implications for research on this compound. Thus far, a number of potential uses for the extracted pigment have been identified, ranging from use as a wood stain [3] to textile dye [5-9], with the most exciting opportunities in the field of optoelectronics. All of these new applications, however, require significant quantities of pure xylindein. All strains and growth conditions produced inhomogeneous extracts containing a mix of secondary metabolites, and while this is expected from biological organisms it is not ideal for (opto)electronic research. While some work has been conducted to begin to identify interfering compounds [12], and a method has been established to use centrifugal partition chromatography for purification [27], more research is required to produce large amounts of pure pigment required for industrial adoption of any technologies built on xylindein. Production of larger amounts of pigment will allow for development of other purification techniques

In this study, liquid cultures were shown to be competitive with solid cultures in terms of pigment production, producing more pigment in earlier stages of growth and yielding a purer product. This suggests liquid culture growth may have the most potential for industrial scale-up and should be a focus of future research, especially as these cultures are easier to scale up for industrial production compared to solid state growth. While no differences in pigmentation concentration as measured by color difference $(\Delta \mathrm{E})$ were seen after week 5 of Chlorociboria growth, electronic properties indicated that C. aeruginascens strain UAMH 7614 was capable of producing the highest quality pigment. Future work with this strain should allow for the most efficient production of xylindein for further optoelectronic research. In addition, the knowledge that large differences exist between xylindein quality of different species and strains will drive future research into collection and identification of new strains that may perform even better. Production of quality xylindein that is straightforward to purify will allow for an expansion of research and potential technological adoption of xylindein into commercial green electronics fields. 


\section{Conclusions}

Previous work on Chlorociboria growth has primarily focused on comparisons of culture size and descriptions of pigmentation with limited comparisons of quantity of extractable xylindein. This study compared previous xylindein production research, and identified Chlorociboria aeruginosa grown under shaking conditions in bacteriological-grade malt as producing the largest mean color difference $(\Delta \mathrm{E})$ of $26.3 \pm 5.9$ in comparison to extracted pigment from shaking liquid, static liquid, and woodchip-amended agar plates, a value comparable with pigment produced in woodchip plates. Higher levels of pigmentation were seen in shaking liquid cultures in early weeks of growth, producing color values matching the standard concentration used in previous publications after only 5 weeks, instead of the normal 2 to 3 months. This result has great implications for industrial pigment production, as liquid cultures have more straightforward scalability.

In addition, pigment production in $C$. aeruginosa and $C$. aeruginascens cultures was seen to be equivalent in color change $(\Delta \mathrm{E})$ of extracted pigment after 5 weeks of growth, with earlier weeks showing variation based more on strain than on species. This may represent significant conservation of pigment metabolic pathway stimulation. C. aeruginascens strain UAMH 7614 was shown to have improved electronic properties, suggesting fewer contaminating secondary metabolites, which makes it preferred for future work on xylindein-based (opto)electronic devices. Identification of other strains and species that may outperform strains tested within this paper may further improve pigment production.

This identification of ideal xylindein production by Chlorociboria spp. in laboratory settings should allow for improved xylindein production and expansion of new research into its use. With larger quantities of xylindein available, methods of purification can be developed to replace fungal extracts containing mixes of compounds with pure pigment for study. This will support further development of this promising organic semiconductor. Technologies using the electronic capabilities of xylindein could support green energy production through organic photovoltaics based on the pigment, as well as other green technologies for a more sustainable world.

Supplementary Materials: The following are available online at http://www.mdpi.com/2227-9717/8/11/1477/s1: Figure S1: Investigations into pigment production of Chlorociboria sp. showing a lack of comparable results due to testing condition differences. Table S1: Colony radius for Chlorociboria aeruginosa on different media, with and without woodchips.

Author Contributions: Conceptualization, R.C.V.C. and S.C.R.; methodology, R.C.V.C.; formal analysis, R.C.V.C.; investigation, R.C.V.C. and G.G.; writing-original draft R.C.V.C., G.G., and S.C.R.; writing-review and editing, S.C.R. and O.O.; visualization, R.C.V.C. and G.G.; supervision, S.C.R.; project administration, S.C.R. and O.O.; funding acquisition, S.C.R. and O.O. All authors have read and agreed to the published version of the manuscript.

Funding: This research was funded by the National Science Foundation "Energy for Sustainability" program (CBET-1705099).

Acknowledgments: Authors would like to thank Linus Pauling Core Laboratories at Oregon State University for their analysis of major sugars in honey and malt samples, and Ariel Muldoon for statistical support.

Conflicts of Interest: The authors declare no conflict of interest.

\section{References}

1. Vega Gutierrez, T.P.; Robinson, C.S. Determining the Presence of Spalted Wood in Spanish Marquetry Woodworks of the 1500s through the 1800s. Coatings 2017, 7, 188. [CrossRef]

2. Blanchette, R.A.; Wilmering, A.M.; Baumeister, M. The use of green-stained wood caused by the fungus Chlorociboria in Intarsia masterpieces from the 15th century. Holzforschung 1992, 46, 225-232. [CrossRef]

3. Robinson, S.C.; Hinsch, E.; Weber, G.; Leipus, K.; Cerney, D. Wood Colorization through Pressure Treating: The Potential of Extracted Colorants from Spalting Fungi as a Replacement for Woodworkers' Aniline Dyes. Materials 2014, 7, 5427-5437. [CrossRef] [PubMed]

4. Robinson, S.C.; Vega Gutierrez, S.M.; Garcia, R.A.C.; Iroume, N.; Vorland, N.R.; Andersen, C.; de Oliveira Xaxa, I.D.; Kramer, O.E.; Huber, M.E. Potential for fungal dyes as colorants in oil and acrylic paints. J. Coat. Technol. Res. 2018, 15, 845-849. [CrossRef] 
5. Palomino Agurto, E.M.; Vega Gutierrez, M.S.; Chen, H.-L.; Robinson, C.S. Wood-Rotting Fungal Pigments as Colorant Coatings on Oil-Based Textile Dyes. Coatings 2017, 7, 152. [CrossRef]

6. Hinsch, E.M. A Comparative Analysis of Extracted Fungal Pigments and Commercially Available Dyes for Colorizing Textiles. Ph.D. Thesis, Oregon State University, Corvallis, OR, USA, 2015.

7. Hinsch, E.M.; Weber, G.; Chen, H.-L.; Robinson, S.C. Colorfastness of Extracted Wood-staining Fungal Pigments on Fabrics: A new potential for textile dyes. J. Text. Appar. Technol. Manag. 2015, 9. [CrossRef]

8. Weber, G.; Chen, H.-L.; Hinsch, E.; Freitas, S.; Robinson, S. Pigments extracted from the wood-staining fungi Chlorociboria aeruginosa, Scytalidium cuboideum, and S. ganodermophthorum show potential for use as textile dyes. Coloration Technol. 2014, 130, 445-452. [CrossRef]

9. Hinsch, E.; Robinson, S. Comparing Colorfastness to Light of Wood-Staining Fungal Pigments and Commercial Dyes: An Alternative Light Test Method for Color Fastness. Coatings 2018, 8, 189. [CrossRef]

10. Giesbers, G.; Van Schenck, J.; Vega Gutierrez, M.S.; Robinson, S.; Ostroverkhovaa, O. Fungi-Derived Pigments for Sustainable Organic (Opto)Electronics. MRS Adv. 2018, 3. [CrossRef]

11. Giesbers, G.; Van Schenck, J.; Quinn, A.; Van Court, R.; Vega Gutierrez, S.M.; Robinson, S.C.; Ostroverkhova, O. Xylindein: Naturally Produced Fungal Compound for Sustainable (Opto)electronics. ACS Omega 2019, 4, 13309-13318. [CrossRef]

12. Giesbers, G.; Krueger, T.; Schenck, J.V.; Court, R.V.; Moore, J.; Fang, C.; Robinson, S.; Ostroverkhova, O. Fungi-derived xylindein: Effect of purity on optical and electronic properties. MRS Adv. 2019, 4, 1769-1777. [CrossRef]

13. Harrison, R.; Quinn, A.; Weber, G.; Johnson, B.; Rath, J.; Remcho, V.; Robinson, S.; Ostroverkhovaa, O. Fungi-derived pigments as sustainable organic (opto)electronic materials. In Organic Photonic Materials and Devices XIX; International Society for Optics and Photonics: Bellingham, WA, USA, 2017; Volume 10101, p. $101010 \mathrm{U}$.

14. Giles, R.G.F.; Green, I.R.; Hugo, V.I. Model studies towards xylindein precursors. S. Afr. J. Chem. 1990, 48, 28-33.

15. Giles, R.G.F.; Reuben, M.K.; Roos, G.H.P. A quinonoid napthtopyranone as a model for the synthesis of the pigment xylindeine. Photochemical formation of the lactone ring. S. Afr. J. Chem. 1979, 32, 127-129.

16. Donner, C.D.; Cuzzupe, A.N.; Falzon, C.L.; Gill, M. Investigations towards the synthesis of xylindein, a blue-green pigment from the fungus Chlorociboria aeruginosa. Tetrahedron 2012, 68, 2799-2805. [CrossRef]

17. Robinson, S.C.; Tudor, D.; Snider, H.; Cooper, P.A. Stimulating growth and xylindein production of Chlorociboria aeruginascens in agar-based systems. AMB Express 2012, 2, 1-7. [CrossRef]

18. Dixon, J.R. Chlorosplenium and its segregates. The genera chlorociboria and chlorencoelia. Mycotaxon 1975, 1, 193-237.

19. Fenwick, G.A. Chlorociboria aeruginascens in laboratory culture. Mycologist 1993, 7, 172-175. [CrossRef]

20. Frenzel, W. Ernährung und Farbstoffbildung von Chlorosplenium aeruginosum ( $O$ ed.); Hölder-Pichler-Tempsky: Vienna, Austria, 1928; pp. 717-746.

21. Stange, S.; Steudler, S.; Delenk, H.; Werner, A.; Walther, T.; Wagenführ, A. Influence of the Nutrients on the Biomass and Pigment Production of Chlorociboria aeruginascens. J. Fungi 2019, 5, 40. [CrossRef]

22. Tudor, D.; Margaritescu, S.; Sánchez-Ramírez, S.; Robinson, S.C.; Cooper, P.A.; Moncalvo, J.M. Morphological and molecular characterization of the two known North American Chlorociboria species and their anamorphs. Fungal Biol. 2014, 118, 732-742. [CrossRef]

23. Robinson, S.C.; Hinsch, E.; Weber, G.; Freitas, S. Method of extraction and resolubilisation of pigments from Chlorociboria aeruginosa and Scytalidium cuboideum, two prolific spalting fungi. Coloration Technol. 2014, 130, 221-225. [CrossRef]

24. Weber, G.; Boonloed, A.; Naas, K.M.; Koesdjojo, M.T.; Remcho, V.T.; Robinson, S.C. A method to stimulate production of extracellular pigments from wood-degrading fungi using a water carrier. Curr. Res. Environ. Appl. Mycol. 2016, 6, 218-230. [CrossRef]

25. Stange, S.; Steudler, S.H.; Delenk, H.; Stange, R.; Werner, A.; Walther, T.; Bley, T.; Wagenfuhr, A. Optimierung der Pigmentbildung vom holzverfärbenden Pilz Chlorociboria aeruginascens-Teil 1: Biomasse- und Pigmentbildung auf Agar und in Flüssigmedien. Holtztechnologie 2018, 59, 52-60.

26. Stange, S.; Steudler, S.; Delenk, H.; Stange, R.; Werner, A.; Walther, T.; Bley, T.; Wagenfuhr, A. Optimierung der Pigmentbildung von dem holzverfärbenden Pilz Chlorociboria aeruginascens Teil 2: Pigmentbildung im Holzsubstrat. Holtztechnologie 2018, 59, 47-54. 
27. Boonloed, A.; Weber, G.L.; Ramzy, K.M.; Dias, V.R.; Remcho, V.T. Centrifugal partition chromatography: A preparative tool for isolation and purification of xylindein from Chlorociboria aeruginosa. J. Chromatogr. A 2016, 1478, 19-25. [CrossRef] [PubMed]

28. Liu, D.; Wang, H.; Park, J.S.; Hur, J.-S. The Genus Chlorociboria, Blue-Green Micromycetes in South Korea. Mycobiology 2017, 45, 57-63. [CrossRef] [PubMed]

29. Ramamurthi, C.S.; Korf, R.P.; Batra, L.R. A revision of the North American species of Chlorociboria (Sclerotiniaceae). Mycologia 1957, 49, 854-863. [CrossRef]

30. Vega Gutierrez, M.S.; Hazell, K.K.; Simonsen, J.; Robinson, C.S. Description of a Naphthoquinonic Crystal Produced by the Fungus Scytalidium cuboideum. Molecules 2018, 23, 1905. [CrossRef] [PubMed]

31. Vega Gutierrez, P.; Almurshidi, B.; Huber, M.; Andersen, C.; Van Court, R.C.; Robinson, S.C. Understanding color vatiation in the pigment produced by Scytalidium ganodermophthorum for artistic applications. International Wood Products 2020. [CrossRef]

32. Johnston, P.R.; Park, D. Chlorociboria(Fungi, Helotiales) in New Zealand. New Zealand J. Bot. 2005, 43, 679-719. [CrossRef]

33. De Carvalho, J.C.; Oishi, B.O.; Pandey, A.; Soccol, C.R. Biopigments from Monascus: Strains selection, citrinin production and color stability. Braz. Arch. Biol. Technol. 2005, 48, 885-894. [CrossRef]

34. Campoy, S.; Rumbero, A.; Martín, J.F.; Liras, P. Characterization of an hyperpigmenting mutant of Monascus purpureus IB1: Identification of two novel pigment chemical structures. Appl. Microbiol. Biotechnol. 2006, 70, 488-496. [CrossRef] [PubMed]

35. Jongrungruangchok, S.; Kittakoop, P.; Yongsmith, B.; Bavovada, R.; Tanasupawat, S.; Lartpornmatulee, N.; Thebtaranonth, Y. Azaphilone pigments from a yellow mutant of the fungus Monascus kaoliang. Phytochemistry 2004, 65, 2569-2575. [CrossRef] [PubMed]

36. Marrocchi, A.; Facchetti, A.; Lanari, D.; Petrucci, C.; Vaccaro, L. Current methodologies for a sustainable approach to $\pi$-conjugated organic semiconductors. Energy Environ. Sci. 2016, 9, 763-786. [CrossRef]

37. Irimia-Vladu, M. “Green” electronics: Biodegradable and biocompatible materials and devices for sustainable future. Chem. Soc. Rev. 2014, 43, 588-610. [CrossRef]

38. Kippelen, B.; Brédas, J.-L. Organic photovoltaics. Energy Environ. Sci. 2009, 2, 251-261. [CrossRef]

39. Dou, L.; You, J.; Hong, Z.; Xu, Z.; Li, G.; Street, R.A.; Yang, Y. 25th Anniversary Article: A Decade of Organic/Polymeric Photovoltaic Research. Adv. Mater. 2013, 25, 6642-6671. [CrossRef]

40. Irimia-Vladu, M.; Głowacki, E.D.; Troshin, P.A.; Schwabegger, G.; Leonat, L.; Susarova, D.K.; Krystal, O.; Ullah, M.; Kanbur, Y.; Bodea, M.A.; et al. Indigo - A Natural Pigment for High Performance Ambipolar Organic Field Effect Transistors and Circuits. Adv. Mater. 2011, 24, 375-380. [CrossRef]

41. Głowacki, E.D.; Leonat, L.; Irimia-Vladu, M.; Schwödiauer, R.; Ullah, M.; Sitter, H.; Bauer, S.; Sariciftci, N.S. Intermolecular hydrogen-bonded organic semiconductors-Quinacridone versus pentacene. Appl. Phys. Lett. 2012, 101, 023305. [CrossRef]

42. Głowacki, E.D.; Tangorra, R.R.; Coskun, H.; Farka, D.; Operamolla, A.; Kanbur, Y.; Milano, F.; Giotta, L.; Farinola, G.M.; Sariciftci, N.S. Bioconjugation of hydrogen-bonded organic semiconductors with functional proteins. J. Mater. Chem. C 2015, 3, 6554-6564. [CrossRef]

43. Anaf, W.; Schalm, O.; Janssens, K.; De Wael, K. Understanding the (in)stability of semiconductor pigments by a thermodynamic approach. Dye. Pigment. 2015, 113, 409-415. [CrossRef]

44. Gsänger, M.; Bialas, D.; Huang, L.; Stolte, M.; Würthner, F. Organic Semiconductors based on Dyes and Color Pigments. Adv. Mater. 2016, 28, 3615-3645. [CrossRef] [PubMed]

45. Tsang, M.P.; Sonnemann, G.; Bassani, D.M. A comparative human health, ecotoxicity, and product environmental assessment on the production of organic and silicon solar cells. Prog. Photovoltaics Res. Appl. 2015, 24, 645-655. [CrossRef]

46. Robinson, S.C.; Gutierrez, S.M.V.; Garcia, R.A.C.; Iroume, N.; Vorland, N.R.; McClelland, A.; Huber, M.E.; Stanton, S. Potential for carrying dyes derived from spalting fungi in natural oils. J. Coat. Technol. Res. 2017, 14, 1107-1113. [CrossRef]

47. Robinson, S.C.; Laks, P.E. Wood species affects laboratory colonization rates of Chlorociboria sp. Int. Biodeterior. Biodegrad. 2010, 64, 305-308. [CrossRef]

48. Stange, S.; Steudler, S.; Delenk, H.; Werner, A.; Walther, T.; Wagenführ, A. Influence of Environmental Growth Factors on the Biomass and Pigment Production of Chlorociboria aeruginascens. J. Fungi 2019, 5, 46. [CrossRef] [PubMed] 
49. Tudor, D.; Robinson, S.C.; Cooper, P.A. The influence of $\mathrm{pH}$ on pigment formation by lignicolous fungi. Int. Biodeterior. Biodegradation 2013, 80, 22-28. [CrossRef]

50. López, J.L.C.; Pérez, J.S.; Sevilla, J.F.; Porcel, E.R.; Chisti, Y. Pellet morphology, culture rheology and lovastatin production in cultures of Aspergillus terreus. J. Biotechnol. 2005, 116, 61-77. [CrossRef]

51. Dronawat, S.N.; Svihla, C.K.; Hanley, T.R. The effects of agitation and aeration on the production of gluconic acid byAspergillus niger. Appl. Biochem. Biotechnol. 1995, 51, 347-354. [CrossRef]

52. Gutierrez, S.M.V.; Van Court, R.C.; Stone, D.W.; Konkler, M.J.; Groth, E.N.; Robinson, S.C. Relationship between Molarity and Color in the Crystal ('Dramada') Produced by Scytalidium cuboideum, in Two Solvents. Molecules 2018, 23, 2581. [CrossRef]

53. Edwards, R.; Kale, N. The structure of xylindein. Tetrahedron 1965, 21, 2095-2107. [CrossRef]

54. Gutierrez, S.M.V.; Gutierrez, P.T.V.; Godinez, A.; Pittis, L.; Huber, M.E.; Stanton, S.; Robinson, S.C. Feasibility of Coloring Bamboo with the Application of Natural and Extracted Fungal Pigments. Coatings 2016, 6, 37. [CrossRef]

Publisher's Note: MDPI stays neutral with regard to jurisdictional claims in published maps and institutional affiliations.

(C) 2020 by the authors. Licensee MDPI, Basel, Switzerland. This article is an open access article distributed under the terms and conditions of the Creative Commons Attribution (CC BY) license (http://creativecommons.org/licenses/by/4.0/). 